\title{
An XMM-Newton survey of the Local Group galaxy M 33 - variability of the detected sources ${ }^{\star, \star \star, \star \star \star}$
}

\author{
Z. Misanovic ${ }^{1}$, W. Pietsch ${ }^{1}$, F. Haberl ${ }^{1}$, M. Ehle ${ }^{2}$, D. Hatzidimitriou ${ }^{3}$, and G. Trinchieri ${ }^{4}$ \\ 1 Max-Planck-Institut für extraterrestrische Physik, Giessenbachstraße, 85741 Garching, Germany \\ e-mail: zdenka@mpe.mpg.de \\ 2 XMM-Newton Science Operations Centre, ESAC, ESA, PO Box 50727, 28080 Madrid, Spain \\ 3 Department of Physics, University of Crete, P/O Box 2208, 71003 Heraklion, Crete, Greece \\ 4 Osservatorio Astronomico di Brera, via Brera 28, 20121 Milano, Italy
}

Received 1 July 2005 / Accepted 14 October 2005

\section{ABSTRACT}

We present an analysis of the individual observations of a deep XMM-Newton survey of the Local Group spiral galaxy M 33. We detected a total of 350 sources with fluxes (in the $0.2-4.5 \mathrm{keV}$ energy band) in the range $6.7 \times 10^{-16}-1.5 \times 10^{-11} \mathrm{erg} \mathrm{s}^{-1}$. This comprehensive study considers flux variability, spectral characteristics, and classification of the detected objects. Thirty-nine objects in our catalogue are new sources, while 311 were already detected in a previous analysis of most of the same data using combined images. We present improved positions of these sources and the X-ray parameters of each source in each individual observation that covers the source. We then used these parameters to systematically search for flux variability on time scales of hours to months or years. The long-term light-curves were generated for the 61 sources showing a significant variability of the (0.2-4.5) keV flux, by a factor of 1.2 to 144 . The detected variability was then used to classify 8 new X-ray binary candidates in M33. Together with the hardness ratio method and cross-correlation with optical, infrared, and radio data, we also classify or confirm previous classification of 25 supernova remnants and candidates, 2 X-ray binaries, and 11 super-soft source candidates ( 7 of which are new SSS candidates). In addition, we classify 13 active galactic nuclei and background galaxies, 6 stars, and 23 foreground star candidates in the direction of M33. Further 206 objects are classified as "hard", approximately half of which are sources intrinsic to M 33. The relative contribution of the classified XRB and SSS in M33 is now comparable to M31. The luminosity distribution of SNRs in both spiral galaxies is almost the same, although the number of the detected SNRs in M 33 remains much higher.

Key words. galaxies: individual: M 33 - X-rays: galaxies - X-rays: binaries

\section{Introduction}

The nearby Sc type galaxy M 33 has been extensively studied in X-rays. Located at a distance of $795 \mathrm{kpc}$ (van den Bergh 1991) and seen under a relatively low inclination angle of $56 \mathrm{deg}$ (Zaritsky et al. 1989), M 33 allows detailed investigation of its source population and diffuse emission from hot gas in the disk.

A total of 17 discrete sources and some diffuse emission of the spiral arms in the vicinity of the nucleus were detected with the Einstein X-ray Observatory (Long et al. 1981; Markert \& Rallis 1983; Trinchieri et al. 1988b). These results were later confirmed with ROSAT HRI and PSPC observations, which

\footnotetext{
* Based on observations obtained with XMM-Newton, an ESA science mission with instruments and contributions directly funded by ESA Member States and NASA.

$\star \star$ Tables 4 and 5 are only available in electronic form at the CDS via anonymous ftp to cdsarc.u-strasbg.fr $(130.79 .128 .5)$ or via http://cdsweb.u-strasbg.fr/cgi-bin/qcat?J/A+A/448/1247 $\star \star \star$ Appendices A and B are only available in electronic form at http://www. edpsciences.org
}

also revealed a number of new point-like sources (Schulman \& Bregman 1995; Long et al. 1996). Combining all ROSAT data, Haberl \& Pietsch (2001) compiled a catalogue of 184 sources in the direction of M 33, and classified the brightest objects using their X-ray properties and identification with optical and radio counterparts.

Pietsch et al. (2004a) (hereafter PMH2004) and Pietsch et al. (2003) have presented the first results of a deep homogeneous XMM-Newton survey of M33. With a sensitivity of $10^{35} \mathrm{erg} \mathrm{s}^{-1}$ in the $0.2-4.5 \mathrm{keV}$ band, this survey is ten times more sensitive than any previous X-ray observation of M 33 . It consists of 24 raster 10-ks observations of 15 overlapping fields covering the whole optical $\mathrm{D}_{25}$ extent of M33 (some fields are re-observed), performed between August 2000 and July 2003. PMH2004 have analysed combined images of the 22 observations made with the medium and thin filters, and detected a total of 408 objects. Having obtained the largest sample of X-ray sources in M 33 to-date, PMH2004 have made use of the broader spectral coverage provided by XMM-Newton to establish criteria for source classification based on hardness 
Table 1. The XMM-Newton M 33 observation log (proposal numbers 010264 and 014198).

\begin{tabular}{|c|c|c|c|c|c|c|c|c|c|c|}
\hline \multirow{3}{*}{$\begin{array}{c}\text { Field } \\
\text { M } 33 \\
(1)\end{array}$} & \multirow{3}{*}{$\begin{array}{c}\text { Obs. ID. } \\
\text { (2) }\end{array}$} & \multirow{3}{*}{$\begin{array}{l}\text { Date } \\
\text { (3) }\end{array}$} & \multirow{2}{*}{\multicolumn{2}{|c|}{$\begin{array}{l}\text { Pointing direction } \\
\text { RA/Dec (J2000) }\end{array}$}} & \multicolumn{2}{|c|}{ EPIC PN } & \multicolumn{2}{|c|}{ EPIC MOS1 } & \multicolumn{2}{|c|}{ EPIC MOS2 } \\
\hline & & & & & \multirow{2}{*}{$\begin{array}{c}\text { Mode }^{+} / \text {Filter } \\
(6) \\
\end{array}$} & \multirow{2}{*}{$\begin{array}{c}T_{\mathrm{exp}}^{\dagger} \\
(7)\end{array}$} & \multirow{2}{*}{$\begin{array}{c}\text { Mode }^{+} / \text {Filter } \\
\text { (8) }\end{array}$} & \multirow{2}{*}{$\begin{array}{l}T_{\exp }^{\dagger} \\
(9)\end{array}$} & \multirow{2}{*}{$\begin{array}{c}\text { Mode }^{+} / \text {Filter } \\
\text { (10) }\end{array}$} & \multirow{2}{*}{$\begin{array}{l}T_{\text {exp }}^{\dagger} \\
(11)\end{array}$} \\
\hline & & & (4) & (5) & & & & & & \\
\hline $1 \mathrm{a}$ & 0102640101 & 2000-08-04 & $1: 33: 51.0$ & $30: 39: 37$ & $\mathrm{FF} /$ medium & 6.09 & PW2/medium & 10.11 & PW2/medium & 9.81 \\
\hline $1 b$ & 0141980501 & 2003-01-22 & $1: 33: 51.0$ & $30: 39: 37$ & $\mathrm{FF} /$ medium & 3.63 & $\mathrm{FF} /$ medium & 7.86 & $\mathrm{FF} /$ medium & 7.86 \\
\hline $1 \mathrm{c}$ & 0141980801 & $2003-02-12$ & $1: 33: 51.0$ & $30: 39: 37$ & $\mathrm{FF} /$ medium & 8.55 & $\mathrm{FF} /$ medium & 10.16 & $\mathrm{FF} /$ medium & 10.16 \\
\hline $2 \mathrm{a}$ & 0102640201 & 2000-08-04 & $1: 34: 40.0$ & $30: 57: 48$ & $\mathrm{EFF} /$ medium & 12.10 & $\mathrm{FF} /$ medium & 16.24 & $\mathrm{FF} /$ medium & 15.94 \\
\hline $3 a$ & 0102640301 & 2000-08-07 & $1: 33: 32.0$ & $30: 52: 13$ & $\mathrm{EFF} /$ medium & 9.10 & $\mathrm{FF} /$ medium & 13.69 & $\mathrm{FF} /$ thin & 13.69 \\
\hline $3 b$ & 0141980401 & 2003-01-24 & $1: 33: 32.0$ & $30: 52: 13$ & $\mathrm{FF} /$ medium & 7.71 & $\mathrm{FF} /$ medium & 11.53 & $\mathrm{FF} /$ medium & 11.64 \\
\hline $4 a$ & 0102640401 & 2000-08-02 & $1: 32: 51.0$ & $30: 36: 49$ & $\mathrm{EFF} /$ thick & 9.20 & $\mathrm{FF} /$ thick & 12.69 & FF/thick & 12.99 \\
\hline $4 \mathrm{~b}$ & 0141980601 & $2003-01-23$ & $1: 32: 51.0$ & $30: 36: 49$ & $\mathrm{FF} /$ medium & 11.38 & $\mathrm{FF} /$ medium & 13.31 & $\mathrm{FF} /$ medium & 13.59 \\
\hline $5 \mathrm{a}$ & 0102640501 & 2001-07-05 & 1:33:02.0 & $30: 21: 24$ & $\mathrm{FF} /$ medium & 9.26 & $\mathrm{FF} /$ medium & 11.76 & $\mathrm{FF} /$ medium & 11.76 \\
\hline $6 a$ & 0102640601 & 2001-07-05 & $1: 34: 08.0$ & $30: 46: 06$ & $\mathrm{FF} /$ medium & 6.56 & $\mathrm{FF} /$ medium & 11.76 & $\mathrm{FF} /$ medium & 11.76 \\
\hline $6 \mathrm{~b}$ & 0141980301 & $2003-07-25$ & 1:34:08.0 & $30: 46: 06$ & $\mathrm{FF} /$ medium & 9.52 & $\mathrm{FF} /$ medium & 11.20 & $\mathrm{FF} /$ medium & 11.50 \\
\hline $7 \mathrm{a}$ & 0102640701 & 2001-07-05 & $1: 34: 10.0$ & $30: 27: 00$ & $\mathrm{FF} /$ medium & 6.86 & $\mathrm{FF} /$ medium & 11.40 & $\mathrm{FF} /$ medium & 11.39 \\
\hline $8 a$ & 0102640801 & 2001-07-07 & $1: 34: 51.0$ & $30: 42: 22$ & $\mathrm{FF} /$ medium & - & $\mathrm{FF} /$ medium & 2.54 & $\mathrm{FF} /$ medium & 2.55 \\
\hline $8 b$ & 0102642001 & 2001-08-15 & $1: 34: 51.0$ & $30: 42: 22$ & $\mathrm{FF} /$ medium & 8.65 & $\mathrm{FF} /$ medium & 11.46 & $\mathrm{FF} /$ medium & 11.46 \\
\hline $9 a$ & 0102640901 & 2001-07-07 & 1:34:04.0 & $30: 57: 25$ & $\mathrm{FF} /$ medium & 7.31 & $\mathrm{FF} /$ medium & 12.51 & $\mathrm{FF} /$ medium & 12.51 \\
\hline $9 b$ & 0141980201 & 2003-07-11 & 1:34:04.0 & $30: 57: 25$ & $\mathrm{FF} /$ medium & 12.14 & $\mathrm{FF} /$ medium & 15.57 & $\mathrm{FF} /$ medium & 15.57 \\
\hline $10 \mathrm{a}$ & 0102641001 & 2001-07-08 & 1:33:07.0 & $30: 45: 02$ & $\mathrm{FF} /$ medium & 4.06 & $\mathrm{FF} /$ medium & 8.74 & $\mathrm{FF} /$ medium & 8.73 \\
\hline $10 \mathrm{~b}$ & 0141980101 & 2003-07-11 & 1:33:07.0 & $30: 45: 02$ & $\mathrm{FF} /$ medium & 9.68 & $\mathrm{FF} /$ medium & 6.81 & $\mathrm{FF} /$ medium & 6.84 \\
\hline $11 \mathrm{a}$ & 0102641101 & 2001-07-08 & $1: 32: 46.0$ & $30: 28: 19$ & $\mathrm{FF} /$ medium & 7.50 & $\mathrm{FF} /$ medium & 10.50 & $\mathrm{FF} /$ medium & 10.20 \\
\hline $12 \mathrm{a}$ & 0102641201 & 2000-08-02 & $1: 33: 38.0$ & $30: 21: 49$ & EFF/thick & 11.30 & $\mathrm{FF} /$ thick & 3.07 & FF/thick & 2.75 \\
\hline $12 b$ & 0141980701 & $2003-01-24$ & $1: 33: 38.0$ & $30: 21: 49$ & $\mathrm{FF} /$ medium & 7.48 & $\mathrm{FF} /$ medium & 5.40 & $\mathrm{FF} /$ medium & 5.40 \\
\hline $13 \mathrm{a}$ & 0102642101 & $2002-01-25$ & $1: 34: 34.0$ & $30: 34: 11$ & $\mathrm{FF} /$ medium & 9.96 & $\mathrm{FF} /$ medium & 12.27 & $\mathrm{FF} /$ medium & 12.27 \\
\hline $14 \mathrm{a}$ & 0102642201 & $2002-01-25$ & $1: 34: 56.0$ & $30: 50: 52$ & $\mathrm{FF} /$ medium & 11.60 & $\mathrm{FF} /$ medium & 13.87 & $\mathrm{FF} /$ medium & 13.87 \\
\hline $15 \mathrm{a}$ & 0102642301 & $2002-01-27$ & $1: 33: 33.0$ & $30: 33: 07$ & $\mathrm{FF} /$ medium & 10.00 & $\mathrm{FF} /$ medium & 12.27 & $\mathrm{FF} /$ medium & 12.27 \\
\hline
\end{tabular}

Notes: ${ }^{+}$FF: full frame; EFF: extended full frame; PW2: partial window $2 .{ }^{\dagger}$ Exposure time in units of ks after background screening to produce the maximum signal-to-noise ratio (see text).

ratios. Applying this method, together with cross-correlations with optical and radio catalogues, PMH2004 were able to classify approximately 350 of the detected X-ray sources.

In this paper we present the results of analysis applied to all 24 XMM-Newton observations of M33 individually, using a less stringent background rejection than in PMH2004 and a more accurate description of the Point Spread Function (PSF). Since our data span a period of almost three years, we were able to study long-term X-ray flux variability of our sample and to use it, together with other methods, to constrain the nature of the detected sources. In addition, we detected new sources in the individual images, and present more accurate X-ray positions of the majority of the sources already detected in the combined data. The paper is organised as follows: in Sect. 2 we present XMM-Newton EPIC observations and data reduction techniques; Sect. 3 describes how the new catalogue was produced; Sects. 4 and 5 present the catalogue and the images of the individual observations. We compare the new catalogue with the combined data catalogue of PMH2004 in Sect. 6, and also cross-correlate it with optical, infrared and radio catalogues (Sect. 7). Section 8 summarises statistical properties of the variable X-ray source population in M33. Finally, classification and properties of the detected $\mathrm{X}$-ray sources, including their spatial and luminosity distribution, are presented in Sect. 9, while Sect. 10 summarises our results. We also include the full band images of all 24 observations showing the detected sources (Appendix A) and longterm light-curves of all variable sources detected in our sample (Appendix B).

\section{Observations and data reduction}

We present analysis of all 24 archival raster XMM-Newton (Jansen et al. 2001) observations of M33. The data analysis was performed using the XMM-Newton Science Analysis System (SAS) v6.0, IDL v6.0, EXSAS/MIDAS 1.4, and FTOOLS v5.3 software packages, the imaging applications DS9 v3.0b6 and KVIS v0.0.11 and the count rate simulator W3PIMMS v3.6a. Most observations were obtained in the Full Frame mode and medium filter, two with the thick filter, and only one exposure of EPIC MOS2 was obtained with the thin filter, as summarised in Table 1 . Table 1 also lists the observation ID and date, and the exposure times for EPIC PN (Strüder et al. 2001), MOS1, and MOS2 (Turner et al. 2001) 


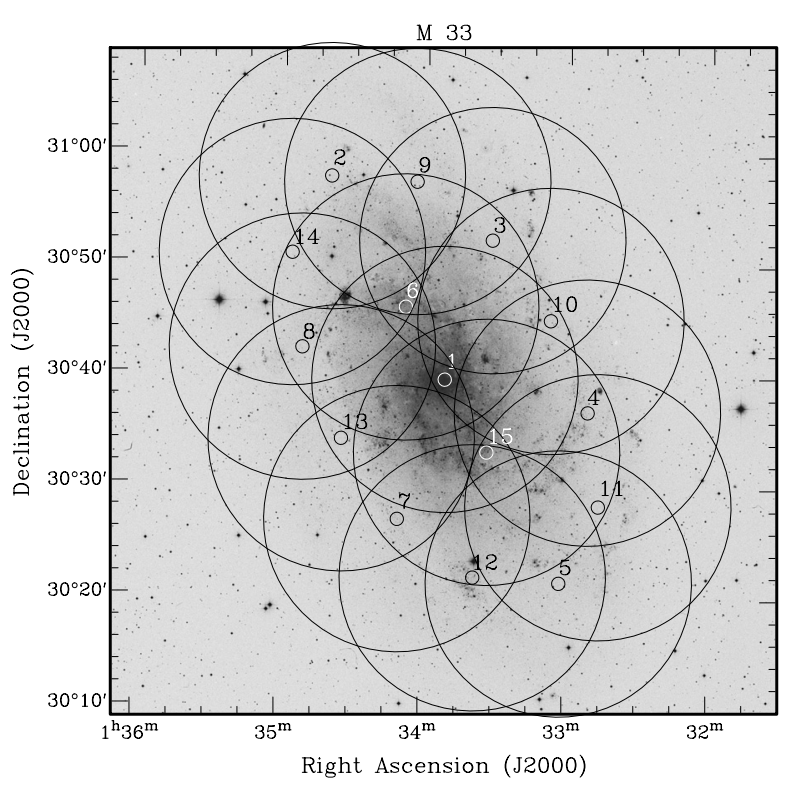

Fig. 1. The XMM-Newton EPIC observation fields overlaid on an optical (DSS-2 red) image of M33. The fields of view (FoV) are approximated by circles of $15^{\prime}$ radius centred at the positions given in Table 1.

that we have obtained after cleaning for episodes of high background, as we explain later. Figure 1 shows the location of the observed fields overlayed on an optical (DSS-2) image of M 33.

For each pointing we produced PN, MOS1, and MOS2 event files using the SAS tasks epchain and emchain, and then manually flagged the remaining bad CCD pixels, which we recognised visually on the images but could not clean with the standard processing. The calibration index files appropriate for the times of observations were generated using the SAS task cifbuild. To optimise the source detection in each field, we then selected the good time intervals (GTIs), which produced the maximum signal-to-noise ratio $(\mathrm{S} / \mathrm{N})$ in each exposure. The maximum $\mathrm{S} / \mathrm{N}$ was calculated using the formula: $S / N \sim t_{\exp } /\left(R_{\mathrm{bkg}}\right)^{1 / 2}$, where $t_{\exp }$ is the total exposure time after the background screening, and $R_{\mathrm{bkg}}$ is the remaining background rate in the image. Such a selection of GTIs results in longer exposure times than those used in PMH2004, since there they had to apply a homogeneous, hence more restrictive, criterion for high background rejection. In addition, PMH2004 had to exclude the (7.2-9.2) keV energy range in order to avoid background variability caused by strong fluorescence lines (Freyberg et al. 2004). When analysing each field individually, however, this was not required.

We produced images from each PN, MOS1, and MOS2 event list in five energy bands: $(0.2-0.5) \mathrm{keV},(0.5-1.0) \mathrm{keV}$, (1.0-2.0) keV, (2.0-4.5) keV, and (4.5-12.0) keV (bands B1 to B5, respectively). We split the standard (0.5-2.0) keV band into two (0.5-1.0 and 1.0-2.0 keV) bands, to be able to apply the hardness ratio method for source classification, introduced by PMH2004. For our PN images in band 1 we selected only single-pixel events (PATTERN $=0$ ), and single and doublepixel events (PATTERN $\leq 4)$ for the other energy bands. We used this PATTERN selection because of the relatively higher noise in the softest PN band. For all five bands of the MOS data, we used 1-4 pixel events (PATTERN $\leq 12$ ), and for each image we produced the corresponding exposure map corrected for vignetting, and also the detector masks to limit the source detection to the detector areas only. These images were then used to run the source detection procedures (described in Sect. 3) separately for each observation.

\section{Production of the catalogue}

\subsection{Source detection}

We run our source detection procedure separately for each pointing, for the 5 energy bands and 3 instruments (in total 15 images) simultaneously, and following the standard method (see e.g. Haberl et al. 2003). Our PN background maps also included the out-of-time (OOT) events, so they did not affect our source detection results. The SAS task emldetect was used to determine the parameters of the detected sources by using a maximum likelihood fit. We selected the likelihood threshold value (DET_ML) of 7 to include a source in the list of the detected sources. As noted by PMH2004, this value was found to be approximately equivalent to the single instrument likelihood of ten, and it corresponds to a 3-4 $\sigma$ detection.

Since the fields of view overlap, we cross-correlated the individual source lists to eliminate multiple detections of the same source. The final position was taken from the observation in which the source has the smallest statistical positional error, which corresponds to the smallest off-axis angle, in most cases.

The positions of the sources common to more than one field were also used to determine the relative astrometry of the observations (see Sect. 3.2). All positions listed in the final catalogue are corrected for that offset.

We determined the X-ray parameters (likelihoods of existence, count rates, fluxes, and hardness ratios) for each source in each individual observation, even when the source is below the detection threshold in that particular observation. This was done by running emldetect again with the final position of each source as an input parameter ("fitposition = no"). By selecting the detection threshold of zero, we obtained the vignetting corrected count rates and corresponding fluxes for each source within the field of view of each observation. For non-detections, we determined a $3 \sigma$ upper limit of the flux.

Finally, to search for extended emission in our sources we repeated the whole procedure optimised for the extended source detection ("fitextent = yes" in emldetect).

\subsection{Source positions}

We determined the relative shifts between our overlapping observations using the positions of the common sources. Sources were considered detected in two different fields if their positions were closer than $3 \times$ the combined statistical errors. We corrected all X-ray positions relative to the observation 0141980801 (1c), since the X-ray source positions in this observation showed the smallest offset with respect to optical and infrared counterparts in our preliminary analysis. Moreover, this field has sources in common with all other fields. 


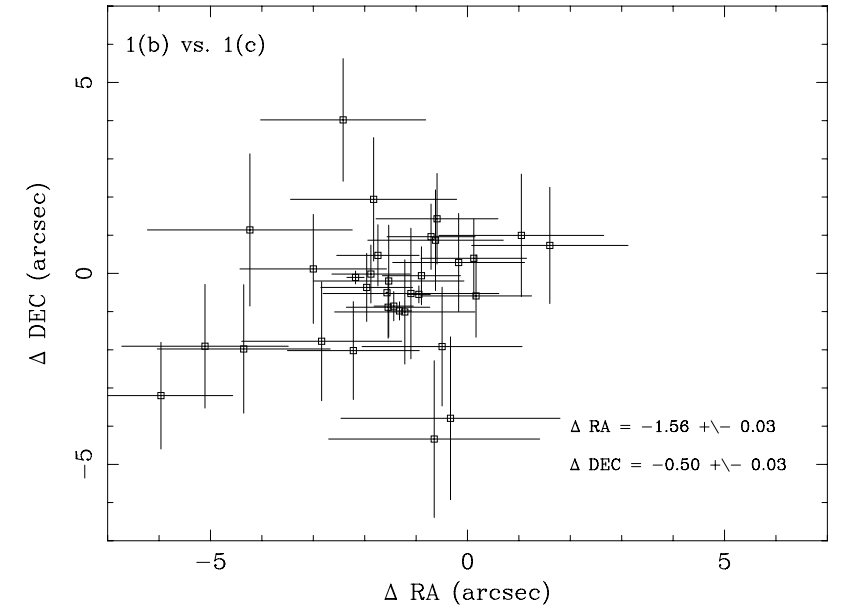

Fig. 2. The RA and Dec offsets of the cross-correlated sources in the observations 0141980501 (1b) and 0141980801 (1c). The error bars represent the combined statistical errors of both X-ray positions. The relative offsets between the two observations are given as the weighted mean of RA and Dec in arcsec.

As one example, we show the RA and Dec offsets of the observation 0141980501 (1b) with respect to the reference observation 0141980801 (1c) in Fig. 2. Similarly, we calculated the average offsets of all observations with respect to our reference pointing (listed in Table 2), and corrected all catalogued $\mathrm{X}$-ray positions.

To determine the systematic errors in the X-ray positions we cross-correlated the final X-ray catalogue with optical (USNO-B1, Monet 1998) and infrared (2MASS, Skrutskie et al. 1997) catalogues. We used a distance of $<3 \times$ the combined X-ray and optical/IR positional error to associate the object to the X-ray source. We identified 113 counterparts in USNO-B1 and 54 in the 2MASS catalogue (Fig. 3), but could not find any significant offset in RA and Dec when compared to either of the catalogues. The $1 \sigma$ residual systematic positional error of 0.5 was then added quadratically to the statistical uncertainties of the detected sources.

\subsection{Hardness ratios, fluxes, and variability factors}

To characterise the spectra of the detected sources, we computed four hardness ratios (HR) for each instrument, defined as HR $i=\left(R_{i+1}-R_{i}\right) /\left(R_{i+1}+R_{i}\right)$, for $i=1$ to 4 , where $R_{i}$ denotes the count rate in band $\mathrm{B} i$. In addition, we calculated the total count rates in each energy band by combining the count rates from all three instruments in a given band and used them to compute the combined hardness ratios. As discussed by PMH2004, due to improved statistics, these combined hardness ratios are useful for source classification.

To convert the vignetting corrected count rates to fluxes in the individual energy bands, we used the energy conversion factors (ECF) for the PN and MOS medium, thin and thick filters calculated using W3PIMMS, taking $100 \%$ of the source counts and assuming a power law spectrum with a photon index of 1.7 absorbed by the Galactic foreground column of $6 \times$ $10^{20} \mathrm{~cm}^{-2}$ (Table 3). The assumed model fits the majority of the
Table 2. The RA and Dec offsets of the M 33 XMM-Newton observations with respect to observation $1 \mathrm{c}$.

\begin{tabular}{|c|c|c|c|}
\hline \multirow{3}{*}{$\begin{array}{c}\text { Field } \\
\text { M 33 } \\
(1)\end{array}$} & \multirow{3}{*}{$\begin{array}{c}\text { Obs. ID. } \\
\text { (2) }\end{array}$} & \multicolumn{2}{|c|}{ Offsets } \\
\hline & & \multirow{2}{*}{$\begin{array}{l}\Delta \mathrm{RA}\left({ }^{\prime \prime}\right) \\
(3)\end{array}$} & \multirow{2}{*}{$\begin{array}{r}\Delta \operatorname{Dec}\left({ }^{\prime \prime}\right) \\
(4)\end{array}$} \\
\hline & & & \\
\hline $1 \mathrm{a}$ & 0102640101 & $-0.38 \pm 0.04$ & $+0.31 \pm 0.04$ \\
\hline $1 b$ & 0141980501 & $-1.56 \pm 0.03$ & $-0.50 \pm 0.03$ \\
\hline $1 \mathrm{c}$ & 0141980801 & - & - \\
\hline $2 \mathrm{a}$ & 0102640201 & $+2.48 \pm 0.09$ & $+0.16 \pm 0.09$ \\
\hline $3 \mathrm{a}$ & 0102640301 & $+1.12 \pm 0.05$ & $+0.25 \pm 0.05$ \\
\hline $3 b$ & 0141980401 & $-0.62 \pm 0.06$ & $+1.89 \pm 0.06$ \\
\hline $4 a$ & 0102640401 & $+1.57 \pm 0.06$ & $-0.54 \pm 0.06$ \\
\hline $4 b$ & 0141980601 & $-1.41 \pm 0.07$ & $+0.00 \pm 0.07$ \\
\hline $5 \mathrm{a}$ & 0102640501 & $-1.11 \pm 0.25$ & $-0.93 \pm 0.25$ \\
\hline $6 a$ & 0102640601 & $+1.44 \pm 0.04$ & $-1.73 \pm 0.04$ \\
\hline $6 b$ & 0141980301 & $+0.93 \pm 0.04$ & $-1.41 \pm 0.04$ \\
\hline $7 \mathrm{a}$ & 0102640701 & $-1.12 \pm 0.07$ & $-1.28 \pm 0.07$ \\
\hline $8 \mathrm{a}$ & 0102640801 & $+0.37 \pm 0.21$ & $-2.29 \pm 0.21$ \\
\hline $8 b$ & 0102642001 & $+1.87 \pm 0.06$ & $-0.74 \pm 0.06$ \\
\hline $9 \mathrm{a}$ & 0102640901 & $-0.54 \pm 0.22$ & $-1.00 \pm 0.22$ \\
\hline $9 b$ & 0141980201 & $-1.32 \pm 0.23$ & $+0.56 \pm 0.23$ \\
\hline $10 \mathrm{a}$ & 0102641001 & $-0.32 \pm 0.05$ & $+0.09 \pm 0.05$ \\
\hline $10 \mathrm{~b}$ & 0141980101 & $-1.28 \pm 0.05$ & $-1.13 \pm 0.05$ \\
\hline $11 \mathrm{a}$ & 0102641101 & $-2.00 \pm 0.24$ & $-0.41 \pm 0.24$ \\
\hline $12 \mathrm{a}$ & 0102641201 & $-0.03 \pm 0.02$ & $-0.41 \pm 0.02$ \\
\hline $12 \mathrm{~b}$ & 0141980701 & $-1.43 \pm 0.17$ & $-0.79 \pm 0.17$ \\
\hline $13 \mathrm{a}$ & 0102642101 & $-0.74 \pm 0.03$ & $-1.08 \pm 0.03$ \\
\hline $14 \mathrm{a}$ & 0102642201 & $+0.54 \pm 0.18$ & $-0.14 \pm 0.18$ \\
\hline $15 \mathrm{a}$ & 0102642301 & $+0.33 \pm 0.12$ & $-0.92 \pm 0.12$ \\
\hline
\end{tabular}

Table 3. Count rate to energy conversion factors for different filters of the EPIC instruments in the energy bands B1-B5, assuming a power law model with a photon index of 1.7 and the galactic foreground absorption of $6 \times 10^{20} \mathrm{~cm}^{-2}$ (Stark et al. 1992).

\begin{tabular}{lrrccrc}
\hline \hline $\begin{array}{l}\text { Detector } \\
\text { EPIC }\end{array}$ & Filter & B1 & $\begin{array}{l}\text { B2 } \\
\left(10^{-12} \mathrm{erg} \mathrm{cm}^{-2} \mathrm{ct}^{-1}\right)\end{array}$ & B3 \\
\hline PN & Medium & 1.03 & 1.10 & 1.71 & 4.90 & 17.07 \\
& Thin & 0.87 & 1.07 & 1.68 & 4.83 & 17.05 \\
& Thick & 1.82 & 1.45 & 1.96 & 5.12 & 17.27 \\
\multirow{2}{*}{ MOS } & Medium & 6.16 & 4.89 & 4.83 & 12.93 & 65.71 \\
& Thin & 5.33 & 4.72 & 4.75 & 12.78 & 65.61 \\
& Thick & 10.36 & 6.34 & 5.53 & 13.50 & 66.61 \\
\hline
\end{tabular}

sources in our sample well i.e. XRBs, AGN, or "hard" sources, while it leads to an underestimation of the flux of soft sources (SNR, foreground stars, SSS) by approximately 10-20\%, or even higher for extremely soft sources like SSS, especially if they are detected only in MOS data (see Table 2 in PMH2004).

To be able to compare our results with the combined data catalogue, we present the total count rates and corresponding fluxes in the "XID" band $(0.2-4.5 \mathrm{keV})$, introduced by 


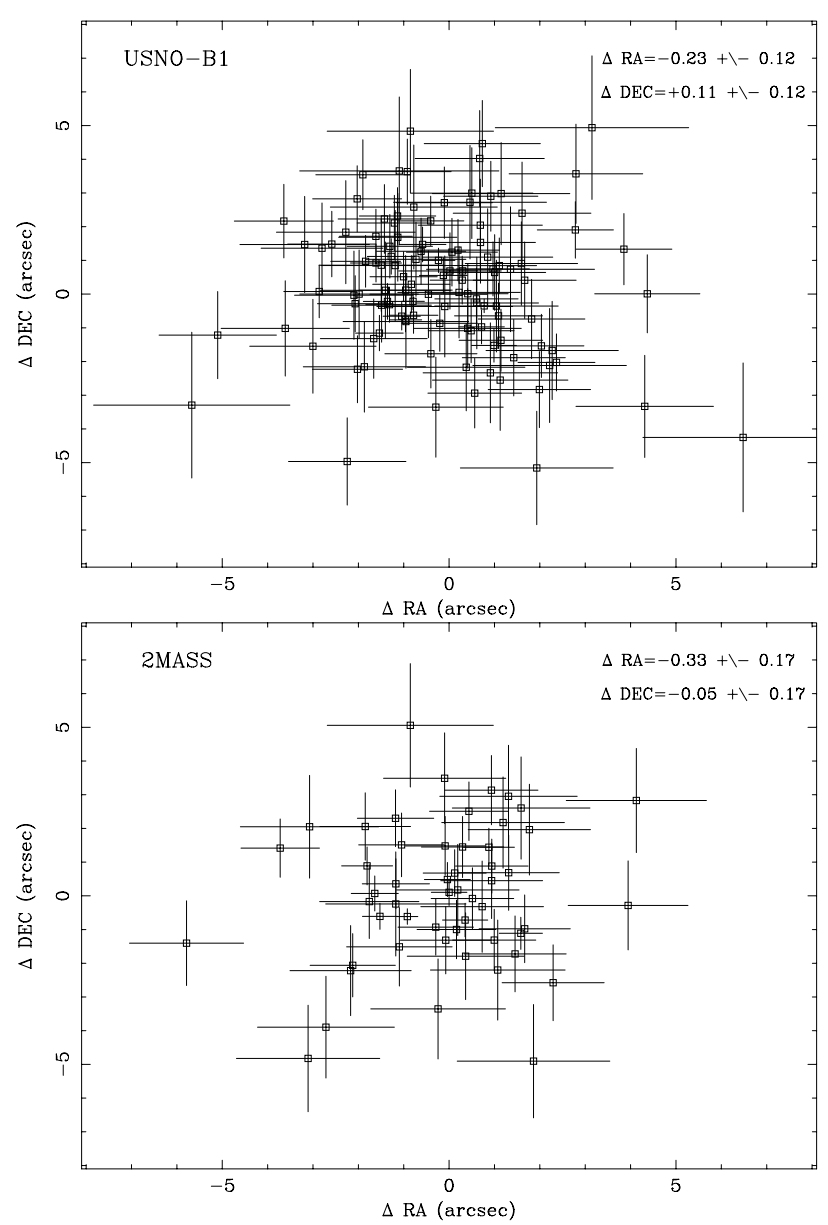

Fig. 3. Upper panel: the RA and Dec offsets of the USNO-B1 counterparts. The error bars represent the combined errors of the statistical error of the X-ray position and corresponding optical source error. The shifts of the X-ray observation with respect to optical data are given as the weighted mean of RA and Dec in arcsec. Lower panel: the RA and Dec offsets of the 2MASS counterparts. The error bars represent the combined errors of the statistical error of the X-ray position and corresponding infrared source error. The shift of the X-ray observation with respect to 2MASS data are given as the weighted mean of RA and Dec in arcsec.

PMH2004. As they note, band B5 suffers from the combined effect of lower sensitivity and a large contamination from the hard background, increasing the noise of all but extremely hard sources.

Since the analysed observations span a period of three years, and are separated by several hours to several months or years, we used them to study X-ray variability on the corresponding time scales. To search for variable sources in our sample, we compared their XID fluxes (or upper limits for nondetections) in different observations. We characterise X-ray variability of each source by its variability factor, defined as $V=F_{\max } / F_{\min }$, where $F_{\max }$ and $F_{\text {min }}$ are the source XID maximum and minimum (or upper limit) flux, respectively. In addition, we calculate a linear significance parameter defined as $S=\left(F_{\max }-F_{\min }\right) / \sqrt{\left(\sigma_{\max }^{2}+\sigma_{\min }^{2}\right)}$, where $\sigma_{\max }^{2}$ and $\sigma_{\min }^{2}$ are the errors of the maximum and minimum flux, respectively (Primini et al. 1993). We define a source as "variable" if
$S>3$. If the minimum XID flux is a non-detection, we use the $3 \sigma$ XID flux upper limit to calculate the lower limit of the variability factor. This is indicated by the variability flag (V_FLAG) value of 1 in Table 4 (V_FLAG $=0$ for detections).

\section{Description of the catalogue}

We detected 350 X-ray sources in the 24 individual observations of the XMM-Newton survey of M 33. Most of the sources in our catalogue have been detected in more than one pointing, which gives us an opportunity to study their X-ray variability (Sect. 8). The results of the survey are summarised in Tables 4 and 5 (available only in electronic form at the CDS).

Table 4 lists the source identification number (Col. 1), source number from the catalogue of PMH2004 when available (Col. 2), and the final source position $(3,4)$ (see Sect. 3.2). The total positional error, which includes the statistical and systematic error of 0.' 5 quadratically summed, is listed in Col. 5, while Cols. 6-10 present the maximum of the detected XID flux and error, variability factor $V$, significance parameter $S$ and variability flag (as described in Sect. 3.3). The remaining columns of Table 4 list the results of the cross-correlation with X-ray, optical, infrared, and radio catalogues, which are described in detail in Sect. 7. Column 11 lists all cross-correlations with sources detected in earlier X-ray surveys of M 33 (Long et al. 1981; Trinchieri et al. 1988b; Schulman \& Bregman 1995; Long et al. 1996; Haberl \& Pietsch 2001), including X-ray variability and other information presented in these catalogues. The detected USNO-B1 counterparts and corresponding X-ray to optical flux ratio (see Sect. 7) are listed in Cols. 12 and 13, respectively, while Cols. 14-17 present 2MASS counterparts and their $J, H$, and $K$-band magnitudes. Columns 18 and 19 list the identified radio counterparts from the NRAO VLA Sky Survey (Condon et al. 1998) and their catalogued $1.4 \mathrm{GHz}$ flux. Column 21 gives all previous source identifications found in the lists of optically and radio selected SNRs (Gordon et al. 1998, 1999), and also in SIMBAD and NED data bases. The final source classification is given in Col. 20, and we discuss it further in Sect. 9.

Table 5 presents the parameters of each source separately for each pointing. Most of the sources are listed in more than one row, each of which summarises the parameters of a single observation. If the source was not detected in a given observation (likelihood of detection $<7$ ) but is included in the FoV, the vignetting corrected upper limits are listed. The columns are: the source number (1), observation (2), likelihood of existence (3), integrated count rate and error $(4,5)$, flux and error in the $0.2-4.5 \mathrm{keV}$ band $(6,7)$, and hardness ratios and errors (8-15), all from the combined PN, MOS1, and MOS2 data. Note that hardness ratios were calculated only for the sources for which the significance of at least one of the contributing count rates is greater than $2 \sigma$. The EPIC PN parameters are listed in Cols. 16-29: exposure (16), likelihood of the source existence (17), count rate and error $(18,19)$, XID flux and error $(20,21)$, and hardness ratios and errors (22-29). The EPIC MOS1 and EPIC MOS2 parameters are listed in the same order in Cols. 30-44 and 45-59, respectively. 


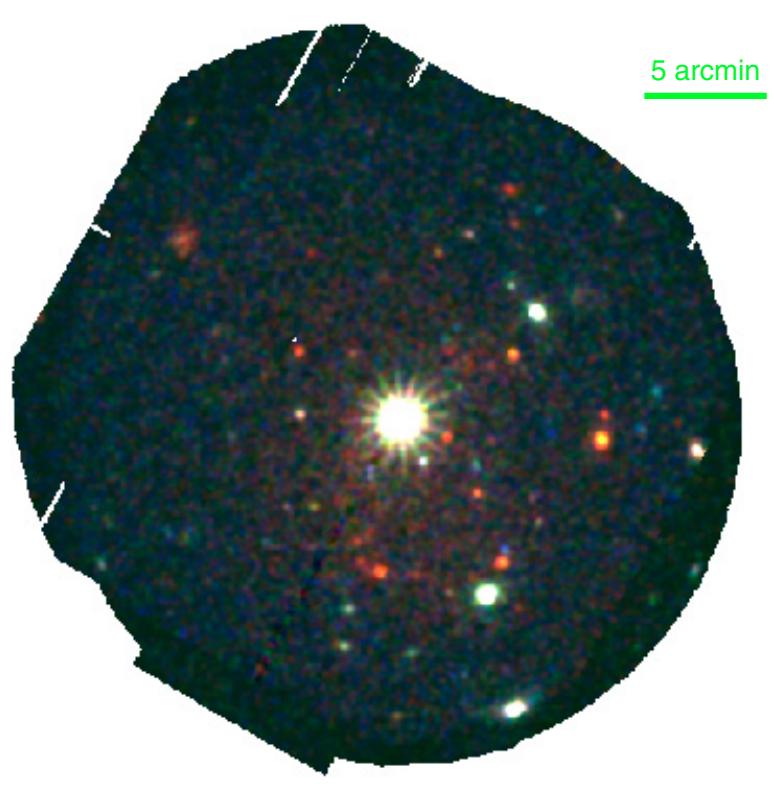

Fig. 4. Linearly-scaled, three-colour XMM-Newton EPIC image of observation 1c combining PN, MOS1, and MOS2 data. Red, green, and blue show, respectively, the $(0.2-1.0) \mathrm{keV}$, (1.0-2.0) $\mathrm{keV}$ and (2.0-12.0) $\mathrm{keV}$ bands. The data in each energy band were smoothed with a Gaussian of FWHM 6". (For a colour image please see the electronic version.)
Sources \#113 and \#254 are detected as extended sources (see Sect. 3.1). Since they are the only two sources with a reliable estimate of the extent parameter in all those fields in which they are detected (extent of $11^{\prime \prime} .2$ and 12'.3 respectively), we list the parameters that were obtained assuming they are extended sources (see details in Sect. 3.1). While several other sources are detected as "extended" in some observations, we believe that their extent is the result of a poor modelling of the PSF at large off-axis angles, and therefore we list them as point sources in the catalogue.

The faintest source (\#264) detected in our sample has the XID flux of $6.7 \times 10^{-16} \mathrm{erg} \mathrm{cm}^{-2} \mathrm{~s}^{-1}$, which is slightly below the sensitivity threshold of the combined data catalogue. However, the source is only detected with PN, and its catalogued flux is calculated as the average flux of all three detectors, and hence reduced. We have only three such faint sources that were detected with PN (\#264, \#212, \#292). The lowest XID flux detected with approximately the same likelihood in all three detectors is $1.8 \times 10^{-15} \mathrm{erg} \mathrm{cm}^{-2} \mathrm{~s}^{-1}$ (\#325). The brightest source, $\mathrm{X}-8$ (\#182), was detected with an XID flux in the range $(1.0-1.5) \times 10^{-11} \mathrm{erg} \mathrm{cm}^{-2} \mathrm{~s}^{-1}$. Sources \#111, \#153, $\# 282$, and \#339, detected in the first run of emldetect when the detection program had fit the source positions, fell slightly below the detectability threshold of 7 in the "fixed position" run of emldetect. This means that the actual positions of these sources are slightly different than our catalogued positions. However, since our detectability threshold is determined in the first run of emldetect, these sources are included in the catalogue. The XID fluxes of the rest of the detected sources are different by less than $5 \%$ in the two runs of emldetect.

\section{Images}

Figures A.1 to A.6 (Appendix A) show the broad band $(0.2-12.0 \mathrm{keV})$ images of all $24 \mathrm{XMM}-$ Newton observations, for PN, MOS1, and MOS2 data combined. The images were smoothed with a Gaussian of FWHM 6", with OOT events excluded from the PN data. The background levels are different in each image, because we cleaned the images to maximise the signal-to-noise ratio in each image individually (see Sect. 2). Onto each image we overlaid the sources detected in that field (big circles), together with the positions of the sources from our catalogue that are covered by that observation but are below the detection threshold (small circles).

Figure 4 illustrates the existence of sources with X-ray colours (i.e. spectra) that are different from the average population; some sources appear red because their X-ray emission is strongest at energies below $1.0 \mathrm{keV}$. Others are blue because they are more prominent in the hard band.

\section{Comparison to the $\mathrm{PMH} 2004$ catalogue}

We cross-correlated our final source list with the combined data catalogue of PMH2004. As expected, the majority of our sources (311) were also detected in the combined images. We also detected 39 sources that are not included in the PMH2004 list. All except 3 of these new sources (\#180, \#208 and \#255) were detected in one field only, either because of relatively higher background in some images, or because these sources are variable or even transient. Several of the sources (e.g. \#32, \#92, \#109, \#140, \#143, \#208) were actually clearly visible in the combined image but too weak or smeared to be picked up by the detection algorithm. Sources \#1 and \#185 were detected near the edges of the two thick filter observations that were not included in the combined image.

With the method described in Sect. 3.2, we were able to improve on the X-ray positions with respect to PMH2004. This was confirmed when we compared ours and the PMH2004 RA and Dec offsets between the X-ray and optical positions for all stars identified as secure optical counterparts, and also for all identified 2MASS counterparts. Our positions were more evenly distributed around a value of 0 than those of PMH2004, which show consistent systematic RA and Dec shifts with respect to optical and infrared data, of approximately -1 " in RA, and $<1^{\prime \prime}$ in Dec. The shifts are within the systematic error of $1.5^{\prime \prime}$ in the combined catalogue determined by PMH2004. 


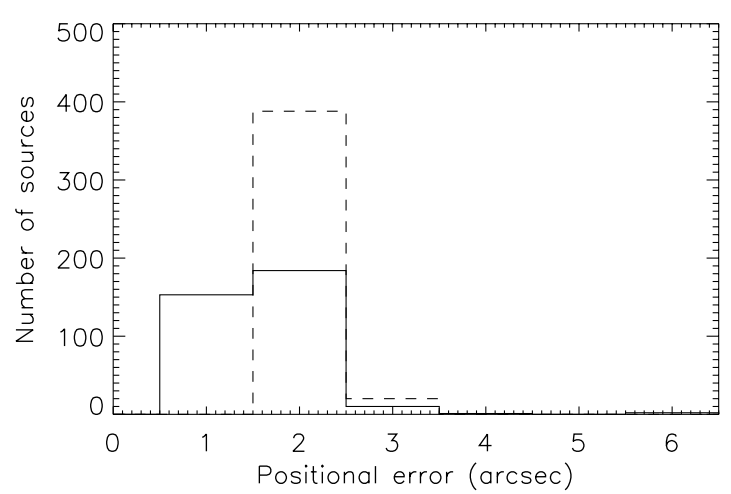

Fig. 5. Histograms of the total positional errors in the individual (full line, this work) and combined (dotted line, PMH2004) data catalogues.

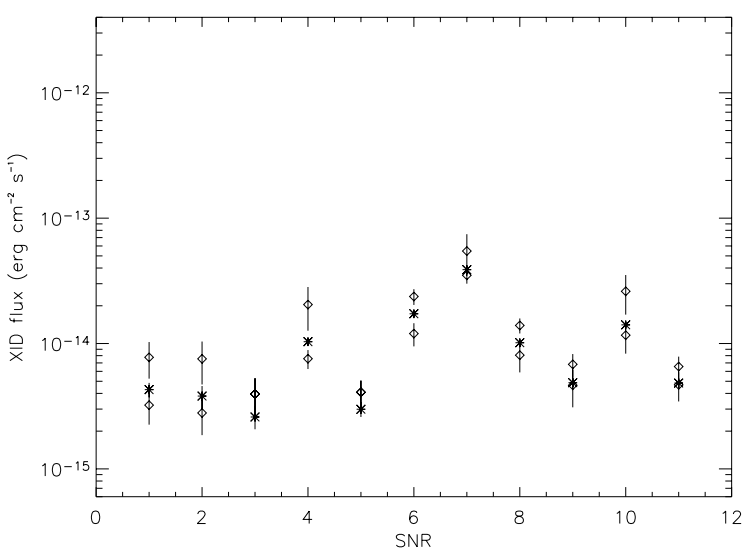

Fig. 6. The maximum and minimum XID fluxes determined in the individual observations (marked as diamonds) for a sample of SNRs detected in both catalogues. The XID fluxes determined in the combined image PMH2004) are marked as asterisks. As expected, SNRs show no significant variability, i.e. the maximum, minimum, and combined catalogue fluxes are within the $3 \sigma$ of the statistical errors.

The smaller systematic error that we estimate for our data also translates into an improved estimate of the total positional errors for most bright sources (Fig. 5).

We compared the XID fluxes and hardness ratios for the 311 sources in common with the PMH2004 catalogue. Figure 6 shows the result of the comparison for SNRs - sources that are expected to show no intrinsic flux variability. For each confirmed SNR and SNR candidate in our data and detected in more than one pointing, we plot the XID flux from PMH2004, and the maximum and minimum XID flux measured in the individual observations. The maximum and minimum flux values are consistent, as for most sources they are within the $\sim 1 \sigma$ of the combined statistical flux errors. A dispersion of up to $2 \sigma$ for a few SNRs is just the result of a normal statistical deviation in a sample of 10-15 measurements. For example, in Fig. 7 we show the measured fluxes in all 13 observations of one such SNRs (SRC \#211). Only one measurement (the maximum) is slightly higher then the rest, but it is determined with a large error, in an observation with short exposure and high background. Hence, the detected variability factor of 2.24 is not significant

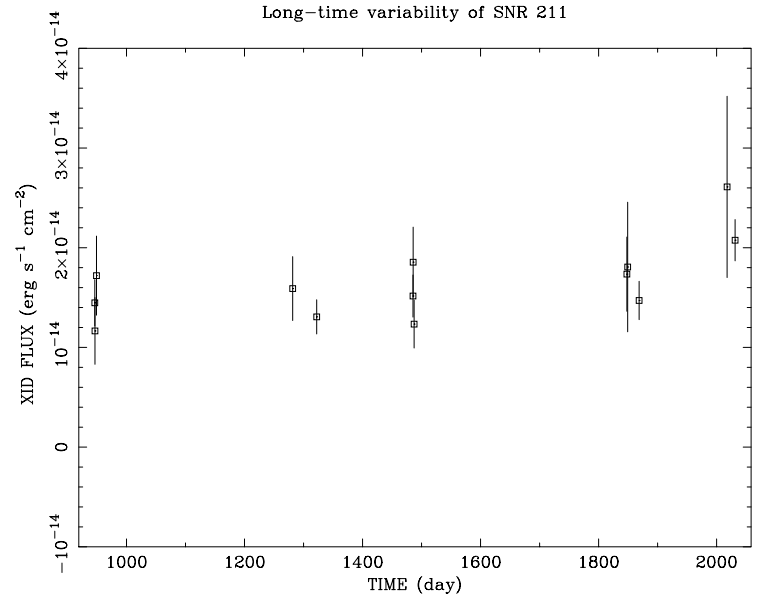

Fig. 7. The long-term X-ray light-curve of an SNR (SRC \#211), detected in 13 individual images. The time is given in days, with the reference day at JD 2450814.5 . The maximum, minimum, and average (PMH2004) flux are also shown in Fig. 6 (SNR number 10). Only the maximum flux deviates slightly from the rest of the measurements. However, its error is large, so the calculated variability factor of 2.24 is not significant $(S=1.49)$.

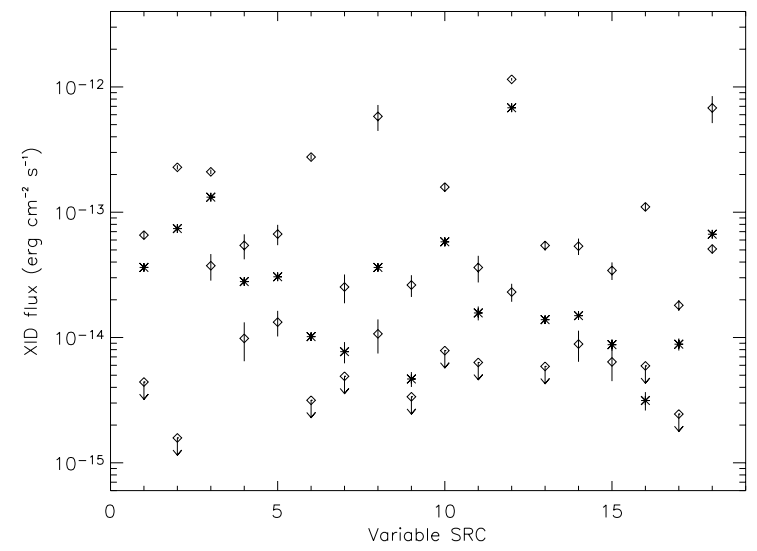

Fig. 8. The maximum and minimum (or upper limit, indicated by a downward arrow) XID fluxes determined in the individual observations (marked as diamonds) for the sample of highly variable sources with the variability amplitude greater than 5 . The asterisks show the XID fluxes determined in the combined image of PMH2004.

$(S=1.49)$. The light-curves of all other SNRs and candidates are similar, confirming their classification.

The situation is different for the sample of highly variable sources (Fig. 8). The flux measured in the combined data is not the intrinsic source flux, but it represents only the averaged high and low state flux measured in the individual images. Its value depends on how many low and high state observations are included in the combined image. For some sources that are in the low state in all or most observations, the combined data flux can even be below the upper limit of the minimum flux determined in individual images.

Due to better statistics, the flux errors in the combined data catalogue are significantly smaller, and hence, the hardness ratios could be more accurately determined for the majority of the detected sources. 
There are 97 sources listed in the PMH2004 catalogue that are below the detection threshold in all individual images and, hence, not detected here. These sources, with fluxes in the range of $1.15 \times 10^{-15} \mathrm{erg} \mathrm{cm}^{-2} \mathrm{~s}^{-1}$ to $1.05 \times$ $10^{-14} \mathrm{erg} \mathrm{cm}^{-2} \mathrm{~s}^{-1}$, were detected in the regions in which the combined image is more sensitive, due to the much longer exposure.

\section{Cross-correlations with other catalogues}

We used the positions listed in Table 4 for a systematic crosscorrelation with X-ray (Haberl \& Pietsch 2001, and references therein), optical (USNO-B1, Monet 1998), infrared (2MASS, Skrutskie et al. 1997), and radio (NRAO VLA Survey, Condon et al. 1998) catalogues, with a radius equal to the $3 \sigma$ error (Col. 5 of Table 4 ) of each source. The results are listed in Table 4. We confirm most of the identifications proposed by $\mathrm{PMH} 2004$, and only comment on a few differences and new entries.

The additional cross-correlations with the ROSAT M33 catalogue of Haberl \& Pietsch (2001) are: HP6, HP68, HP90, HP128 (cross-correlated with new sources not detected in the combined data), and HP64 (a strong source also detected in the combined image but with the position just outside the $3 \sigma$ distance from the ROSAT source).

In most cases we identified the $\mathrm{X}$-ray source with the brightest of the sources in the USNO-B1 catalogue that falls within the search region. We then used the X-ray to optical flux ratio, calculated as $\log \left(\frac{f_{\mathrm{x}}}{f_{\text {opt }}}\right)=\log \left(f_{\mathrm{x}}\right)+\left(m_{\mathrm{B} 2}+m_{\mathrm{R} 2}\right) /(2 \times 2.5)+$ 5.37, following Maccacaro et al. (1988), to distinguish foreground stars from SNRs and AGN (see Sect. 9). For 10 sources in common with PMH2004, namely sources \#8, 61, 81, 84, 90, $105,183,188,220$, and 270 , we identified a different counterpart in the USNO-B1 catalogue, because of our new positions. We also found 7 new identifications (source \#144, 186, $230,245,308,310$, and 343), but did not confirm the identifications of sources \#241, 248 and 278, which are now without a USNO-B1 counterpart. In addition, there are 10 out of the 39 new sources for which we find an optical counterpart in the USNO-B1 catalogue.

PMH2004 did not do a systematic cross-correlation with the 2MASS catalogue (Cutri et al. 2003), but only listed one counterpart that was suggested by SIMBAD. We correlated our catalogue with 2MASS, and found 54 identifications, confirming the PMH2004 identification of the source \#170 (196 in PMH2004).

The cross-correlation with the lists of optically and radio selected SNRs (Gordon et al. 1998, 1999) and SIMBAD and NED data bases allowed us to obtain several new identifications, which are discussed in Sect. 9.

Finally, we detected 6 new radio counterparts in the NRAO VLA Sky Survey (Condon et al. 1998). Note that for some extended radio counterparts the positions of the radio and X-ray sources are separated by $\sim 15-20^{\prime \prime}$.

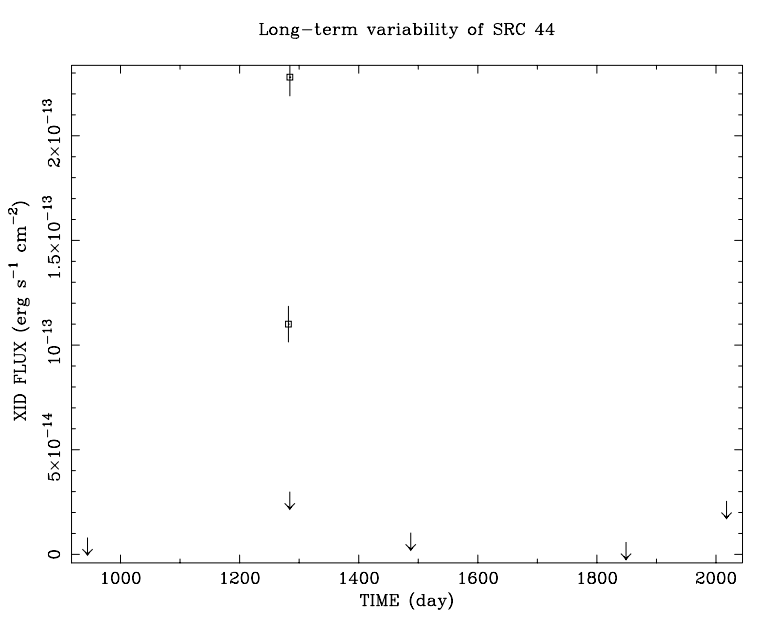

Fig. 9. The long-term X-ray light-curve of source \#44, showing an extreme flux variability by a factor of 144 on a time scale of several days and, hence, classified as an XRB candidate (see Sect. 9). The time is given in days after JD 2450814.5 .

\section{Variability}

We detected significant long-term X-ray flux variability of $61(20 \%)$ out of 301 sources covered by more than one pointing. Only 2 of these sources were known to be variable from previous variability studies of M 33 using Einstein data (Peres et al. 1989). The variability factors ( $V$, see Sect. 3.3), or lower limits detected here and defined as the ratio between the maximum and minimum flux (or upper limit), are in the range 1.2 to 144 , on a time scale of a few hours to several months or years.

The long-term light-curve of the most variable source in our sample is shown in Fig. 9, while the light-curves of the remaining 60 variable sources are shown in Figs. B.1 to B.4 (Appendix B). For each source we plot all measured fluxes in all observations that cover the source, or the $3 \sigma$ upper limits, omitting only a few meaningless upper limits that are higher than the detected fluxes. Such high upper limits were determined only for a few sources in high background images. Some sources were detected in a large number of individual observations, and exhibited large flux variations on a relatively short time scale of (several) hours. In some sources the variability can be described as flares.

The long-term flux variability was used to further constrain the nature of these sources. The variability amplitudes and light curve shapes were used to classify eight relatively hard and luminous sources as XRB candidates, as they were observed changing their XID flux by a factor of ten to more than a hundred (Sect. 9).

We confirm the X-ray flux variability of both of the two previously known XRB - the eclipsing binary X-7 designated here as source \#150 (Pietsch et al. 2004b, and references therein), and the ultra-luminous source near the nucleus $\mathrm{X}-8$ or source \#182 (Dubus et al. 1997; Dubus \& Rutledge 2002; Dubus et al. 2004). Besides XRBs and candidates, the most variable sources detected in our sample are SSS candidates. The sample of the variable sources also includes 6 star candidates, 1 galaxy, 3 AGN candidates, 36 hard, 2 unclassified 

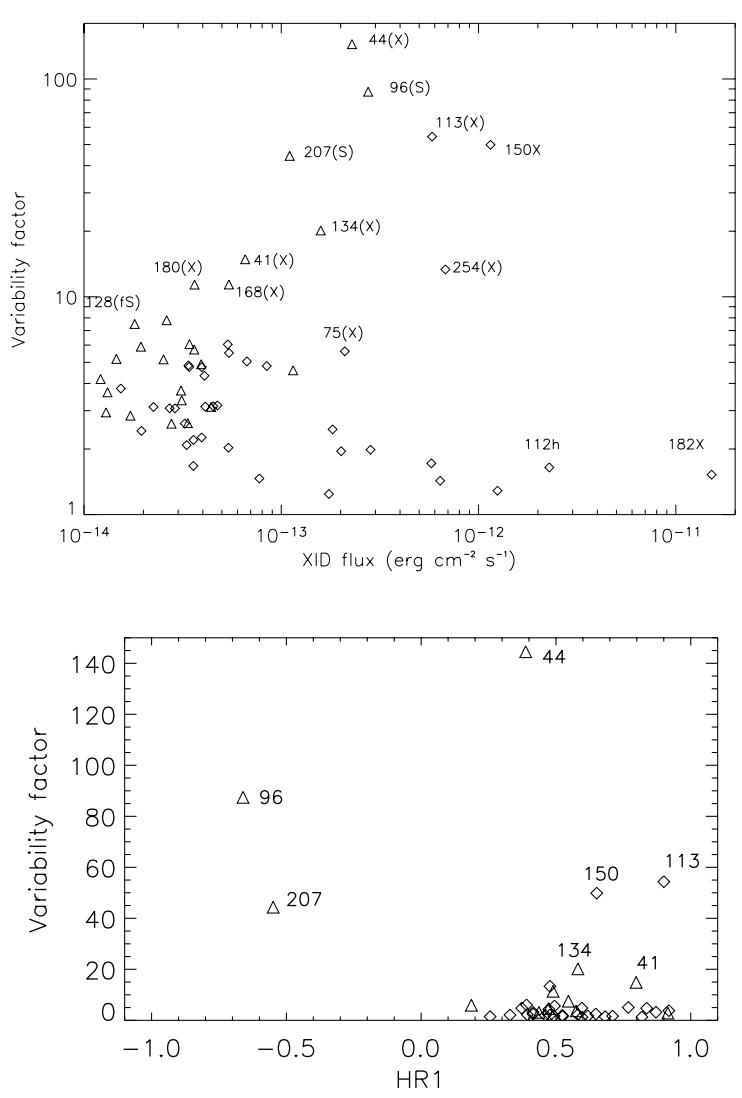

Fig. 10. Upper panel: the variability factor as a function of the maximum XID flux for the 61 significantly variable sources from Table 4. The lower limits of the variability factors are marked as triangles pointing up, while values determined from measured minima are shown as diamonds. For the brightest and most variable sources, the catalogue source number and classification are also marked: $\mathrm{X}$ indicates X-ray binary, S supersoft source, fS foreground star and $h$ "hard" source. The same symbols in brackets denote candidates. Lower panel: the variability factor versus HR1. The lower limits are shown as triangles, while the calculated values are marked as diamonds. The catalogue source numbers are also marked. Sources \#96 and \#207 are classified as SSS candidates, while the rest of the marked sources are XRB or XRB candidates.

sources. Three optically identified SNRs were also found to be variable in our analysis, and we changed the classification of these sources (discussed in detail in Sect. 9.4).

Figure 10 (upper panel) shows the detected variability factor (or lower limit) as a function of the maximum flux, for all variable sources. As already noted, the most variable sources in our sample are XRB and SSS candidates, with an XID high state flux greater than $\sim 1 \times 10^{-13} \mathrm{erg} \mathrm{cm}^{-2} \mathrm{~s}^{-1}$, corresponding to a luminosity of $10^{36}-10^{37} \mathrm{erg} \mathrm{s}^{-1}$ at the distance of M 33 . Such luminosities are expected for X-ray binary systems. Since the faintest sources detected in the individual images have XID fluxes of about $\sim 1 \times 10^{-15} \mathrm{erg} \mathrm{cm}^{-2} \mathrm{~s}^{-1}$, determined with large errors, we could not detect any significant variability for such objects. Hence, the faintest variable source in our sample has an XID high state flux of $1.2 \times 10^{-14} \mathrm{erg} \mathrm{cm}^{-2} \mathrm{~s}^{-1}$. As expected, there is a correlation between the variability factor (and also variability significance) and maximum XID flux, since large flux variations could only be detected for luminous sources. However, as Fig. 10 shows, several highly luminous sources exhibit a small amplitude but highly significant variability (e.g. \#182, 131, 112, 124, 237). The variability factors of these sources are in the range of 1.3 to 1.7 , with the significance as high as 10 to 35 . Clearly such behaviour can only be seen in luminous sources detected with small errors on the flux determination. Most of these sources also showed some indications of variability in previous X-ray observations. As already noted, source \#182 (X-8) is known from previous studies to be variable with small flux modulations. In addition, sources \#131 (the Einstein source X-6, Long et al. 1981; Trinchieri et al. 1988a) and \#112 (X-4) showed flux variations of at least $40 \%$ in a BeppoSAX study, but since the detected variability was comparable to systematic uncertainties, it was regarded as tentative (Parmar et al. 2001).

In order to exclude a possible systematic error in our automatic flux determination, we compared the fluxes of all sources in overlapping fields. For example, we checked whether some observations show systematically higher (or lower) flux for all or most sources within the FoV. However, we could not detect any trends. The maximum and minimum XID fluxes seem to be distributed randomly between the observations, and they are not related to the filter used, or to the off-axis angle of the source. We also note that, although most of the brightest sources in our data show some degree of variability, there are also highly luminous sources (e.g. \#288, 348) that show no significant variability.

The lower panel of Fig. 10 shows the relationships between the variability factor and hardness ratio HR1 determined in the high states. We plot only the sources with hardness ratio errors smaller than 0.2 , and also mark the catalogue source number of the most variable sources. The HR plot shows that the sample of highly variable sources includes SSS and XRB candidates, which occupy two distinct regions on this plot. For example, sources \#96 and 207, classified as SSS candidates, appear on the left hand side, while the rest of the marked sources have much harder spectra, in agreement with their proposed classification as XRB or candidates. We also identified two distinct spectral classes of sources with much smaller variability amplitudes: soft sources with the HR2 in the range -0.8 to -0.5 are most probably foreground stars; and sources with well-defined HR3 and HR4, we classified as "hard".

\section{Classification of the detected sources}

We classified the sources detected in our data into different classes of X-ray emitting objects: foreground stars (fgStar), galaxies (GAL), AGN, super-soft sources (SSS), supernova remnants (SNR), and X-ray binaries (XRB), using the crosscorrelation with catalogues at other wavelengths, together with the X-ray properties determined in this survey. In a few cases we were able to identify an X-ray source with a source already classified from optical, infrared, or radio data. However, in most cases we could not find this kind of information, so it is useful to define criteria to classify sources based on their X-ray characteristics. This is demonstrated by Haberl \& Pietsch (2001), who have used the X-ray hardness ratio diagrams to define regions populated by different classes of X-ray sources, 
using well-studied sources in the Magellanic Clouds (Haberl \& Pietsch 1999). PMH2004 have expanded it to the broader XMM-Newton energy range and applied it successfully to classify more than 350 sources detected in the XMM-Newton combined image of M 33, and also more than 600 sources in M 31 (Pietsch et al. 2005b).

Here we adopt the same classification criteria as defined in Table 5 of PMH2004, and add the information derived from the $\mathrm{X}$-ray variability and near-infrared colour and brightness that we derived in this work. We list our classifications in Col. 20 of Table 4. Similarly to PMH2004, we classify a source only if all criteria are fulfilled; otherwise, the source is a candidate for a particular class of objects (indicated by pointed brackets).

The majority (206) of the detected sources were classified as "hard", using the selection criteria defined by PMH2004 as HR2 - HR2 error $>-0.2$, or only HR3 and HR4 as defined and no other classification. From comparison with the X-ray background surveys (Brandt et al. 2001; Rosati et al. 2002), we estimate that approximately 100-150 of these sources would be background objects - AGN and galaxies - 13 of which we have already identified. There are also 28 foreground stars and star candidates in our catalogue and 54 sources that have relatively soft spectra and that we could not classify. The rest of the detected sources most probably belong to M33 10 XRB and XRB candidates, 25 SNRs and SNR candidates, and 11 SSS candidates.

\subsection{Foreground stars (fgStar)}

We adopted the criteria defined in Table 5 of PMH2004 $\left(\log \left(f_{\mathrm{x}} / f_{\text {opt }}\right)<-1.0, \mathrm{HR} 2<0.3\right.$ and HR3 $\left.<-0.4\right)$ to classify foreground stars, and added near-infrared brightness and colour criteria: $(J \mathrm{mag}-K \mathrm{mag}<0.8)$, and $(J \mathrm{mag}<12.5)$ (Finlator et al. 2000).

Out of the 5 foreground stars and 30 star candidates classified by PMH2004, we confirm the detection and classification of 4 stars plus 21 foreground star candidates. We also classify one of the new sources (\#189) and a previously listed source candidate (\#174) as foreground stars, identified also with SIMBAD stars. The first star is listed in the catalogue of OB associations in the southern arm of M33 and classified on the basis of UBV CCD photometry (Regan \& Wilson 1993). The star classification of the second object is based on the measured proper motion and listed in The Tycho Reference Catalogue (Hog et al. 1998). We classify further 3 objects as foreground star candidates.

Only four stars and candidates do not have a 2MASS counterpart, and one star candidate (\#110) has the infrared brightness and colour slightly out of range. The X-ray spectrum of this object is also found to be slightly harder than expected for a typical star. However, since the optical data confirm the stellar classification and its light-curve (Fig. B.1) resembles a flaring event, we leave the PMH2004 classification for this object.

There are three other foreground star candidates (\#128, $\# 170$, and \#309, see the light curves in Figs. B.2 and B.4) that show significant X-ray variability on the time scale of days to months, probably due to flaring. We also classify \#133 and 186 as foreground stars, because the sources are variable; hence their previous SNR classification was not confirmed (see Sect. 9.4). Only one star candidate (\#170) could not be associated with the USNO-B1 counterpart, although its HRs and infrared brightness and colour confirm the stellar classification. The source is also listed in the catalogue of blue and red giants in M 33 (Ivanov et al. 1993). All of the classified foreground stars and candidates have the X-ray fluxes and hardness ratios in the range expected for this class of objects.

Sources \#263 and \#309 are classified as foreground star candidates by PMH2004. We identified these sources with the same USNO counterparts. However, in our data they have $\log \left(f_{\mathrm{x}} / f_{\text {opt }}\right)>-1-$ outside the range assumed for stars, because we measured a higher X-ray flux (see Sect. 6 for possible explanations on differences in the flux measured in the two catalogues). Since the infrared data does not distinctively confirm the stellar classification of the first source and its spectrum is slightly harder, we leave it without classification. A relatively higher X-ray flux of the second source could be explained as flaring (see Fig. B.4). We keep the stellar classification of this source, because it is also confirmed by its HRs and infrared data.

Sources \#8 and \#11 have been suggested as possible foreground stars by PMH2004 based on their hardness ratios. However, they could not be associated with any optical counterpart. Due to our improved positions we identified the USNO counterparts, but the corresponding $\log \left(f_{\mathrm{x}} / f_{\text {opt }}\right)$ are larger than -1 and do not support the proposed classification. The hardness ratios, which are the same as calculated by PMH2004, classify the first source as an SNR candidate, while we could not classify the second one. According to its spectrum and $\log \left(f_{\mathrm{x}} / f_{\text {opt }}\right) \sim 0$ the source could be either an SNR or a galaxy.

\subsection{Galaxies (GAL) and AGN}

The selection criteria used to classify galaxies and AGN are defined as either a radio source with a relatively hard X-ray spectrum compared to a SNR or an optical identification with a galaxy (Table 5, PMH2004). We detected and confirmed the classification of 11 out of 14 galaxies and AGN, and we classified one more source (\#57) as AGN, because of its hard spectrum and spatial correlation with the NVSS source $013245+303859$. Source \#126 classified by PMH2004 as an SNR or AGN is found to be significantly variable in our data, with flux variations of a factor of 5 on a time scale of several days (Fig. B.2). We classified this source as AGN, as the detected variability would make a SNR classification unlikely.

\subsection{Super-soft sources (SSS)}

Luminous super-soft sources, discovered as a new class in the LMC by ROSAT (Trümper et al. 1991; Greiner et al. 1991), display extremely soft X-ray spectra, which can be represented by a blackbody model with the effective temperature of the emitting object below $50 \mathrm{eV}$. Luminous $\left(10^{36}-10^{38} \mathrm{erg} \mathrm{s}^{-1}\right)$ 
super-soft sources are generally believed to be binary systems with white dwarfs accreting from more massive hydrogenburning donors (van den Heuvel et al. 1992). They have been observed in our own and in nearby galaxies, sometimes showing significant variability on different time-scales, from several days to several months, while some other SSSs have been found to be recurrent transients (Greiner et al. 2004; Di Stefano et al. 2004; Osborne et al. 2001).

The selection criteria for SSSs in the combined XMM-Newton catalogue $(\mathrm{HR} 1<-0.2$, HR2 - HR2 error $<-0.99$ or HR2 not defined, HR3 and HR4 not defined) were derived by calculating the HRs for an assumed 25 and $50 \mathrm{eV}$ black body model (Table 5, PMH2004). We confirm 4 out of 5 SSS candidates. Two (\#96, \#207) are very luminous and also among the most variable sources detected in our data (variability factors of 87 and 44, respectively; Figs. B.1 and B.3), which makes their identification with super-soft accreting binary systems very likely. Super-soft source candidates \#48 and \#285 are slightly less luminous, and we could not detect any significant flux variability in our observations. Note that the fluxes given in Tables 4 and 5 are calculated by assuming a power law spectrum and the galactic foreground absorption as for all other sources. However, due to their significantly different spectrum, we estimate that their fluxes are underestimated by as much as $30-40 \%$.

In addition to these four SSSs, we propose 7 new SSS candidates, detected only in the individual images with luminosities of $\sim 10^{35}-10^{36} \mathrm{erg} \mathrm{s}^{-1}$, at the distance of M 33. Some of these SSS candidates may be low-luminosity objects, such as e.g. single, non-interacting white dwarfs, magnetic cataclysmic variables, or novae several years after outburst (Greiner 2000). Recently, Pietsch et al. (2005a) found that almost all of the 21 X-ray counterparts of optical novae detected in M 33 and M31 were identified as SSS according to their HRs. However, none of the classified SSS sources in this or in the $\mathrm{PMH} 2004$ catalogue is associated with the known optical novae, probably due to the small number of detected optical novae in M33.

\subsection{Supernova remnants (SNR)}

Many SNRs in M 33 were detected and classified in the optical (see Gordon et al. 1998, and references therein) and radio observations (see Gordon et al. 1999, and references therein). PMH2004 have identified a total of 21 of these known SNRs, and their X-ray properties confirm the optical/radio identification as an SNR. Further 23 SNR candidates, fulfilling either the HR criteria (HR1 $>0.1$ and HR2 $<-0.4$ ) or coinciding with a classified radio or optical SNR, are also listed in the combined data catalogue.

We confirm the PMH2004 classification of 14 SNRs plus 11 candidates. We checked that the variability factors of these sources are consistent with no variability (see also Sect. 6). However, for sources \#108, 133, and 186, identified and listed in the M 33 SNR catalogue of Gordon et al. (1998) as SNRs 21, 28 , and 55 , we detected small but significant flux variations.
Source \#108 (SNR 21, Gordon et al. 1998) has an optical extent of approximately $6-7$ arcsec $(28 \mathrm{pc}$ at the distance to M 33). We determined the parameters of this source from our point source run of emldetect (see Sect. 3), because the extended emission was detected only marginally in some observations when we tried to fit it. This could have caused the detected small flux variations with an amplitude of $1.24(S=4.52$, the light-curve is shown at Fig. B.1) in our data. However, some indications for possible variability of this source (the Einstein source X-3, Long et al. 1981; Trinchieri et al. 1988a) were also found by Peres et al. (1989).

Source \#186 (X-13, SNR 55) was reported to have different count rates in two Einstein observations separated by 6 months, although the source was too weak to definitely confirm the detected variability (Peres et al. 1989). We found that \#186 changes its XID flux by a factor of 2.2 (significance 4.12). As its light-curve shows, (Fig. B.3) the source goes through several "high" states with the XID flux of 3-4 $\times$ $10^{-14} \mathrm{erg} \mathrm{cm}^{-2} \mathrm{~s}^{-1}$ and "low" states, when the flux decreases to below $2 \times 10^{-14} \mathrm{erg} \mathrm{cm}^{-2} \mathrm{~s}^{-1}$. In a recent study of SNRs in M 33 with Chandra, Ghavamian et al. (2005) have found this $\mathrm{X}$-ray source to be point-like in Chandra data, which also indicates that the detected X-ray emission is not associated with the optically selected SNR.

Finally, source \#133 (SNR 28, not detected with Einstein) changed its flux by a factor of 1.67 ( $S=3.47$, see Fig. B.2). Ghavamian et al. (2005) report that this X-ray source has a flux, as determined in Chandra observations, that is two times smaller when compared to the PMH2004 XID flux.

A discrepancy between the XID flux measured with XMM-Newton and Chandra was also noted for \#138 (SNR 29), which in our analysis has a variability factor of 1.98 , but determined below the $3 \sigma$ significance level $(S=2.77)$.

While the small variability amplitude of \#108 might be explained by an error in the XID flux determination due to the large extent of this source, this certainly would not be the case for the other two (possibly three) sources. According to their hardness ratios, all four sources could also be classified as stars. Furthermore, we identified the USNO counterparts of \#133 and $\# 186$, and the calculated $\log \left(f_{\mathrm{x}} / f_{\text {opt }}\right)$ is in the range expected for stars. Hence, we classified these two sources as possible foreground stars. We keep the proposed SNR classification for \#138, and leave \#108 without classification. However, further studies are needed to confirm the nature of the X-ray emission, which coincides with these optically selected SNRs.

For one source (\#206) classified in PMH2004 as SNR candidate, we found significant variability (variability factor of almost 6, Fig. B.3). The HRs of this source (in PMH2004 and also in our data) are consistent with both the stellar and SNR classification, but since the source could not be associated with an optical counterpart, PMH2004 list it as an SNR candidate. However, the detected variability in our data is inconsistent with the proposed SNR classification, so we leave this source unclassified.

The giant HII region NGC 604, the brightest optical source in M33, is also identified with an SNR candidate in PMH2004, based on its HRs. The source is detected in our analysis as the extended source \#254. We confirm its HRs but found it to be 
variable, with a variability pattern similar to X-7 (Fig. B.4). Since the source is extended in all observations, we determined its parameters by fitting its extent in emldetect. However, high resolution Chandra archival images show that this complex HII region is resolved into a collection of point sources probably of different natures (SNRs, XRBs), and includes perhaps some diffuse hot gas emission. The same is true for the BCLMP 290 (Boulesteix et al. 1974), another optically bright extended region in M33, which shows a hard X-ray spectrum and significant long-term X-ray variability (source \#113, Fig. B.1). We classify this source as an XRB candidate (see next section).

Finally, we note that Ghavamian et al. (2005) have found optical counterparts of two SNR candidates (\#61, and PMH2004 source 270 not detected in the individual observations) selected using the HR method. The optical data confirms the proposed SNR classification.

\section{5. $X$-ray binaries $(X R B)$}

As discussed by PMH2004, HRs alone cannot be used to distinguish XRBs from AGN or plerions, because these classes of sources occupy the same regions in the HR diagrams. Therefore, PMH2004 introduced "hard" sources as a new class. Both XRBs and AGN are known to be variable. However, XRB in general show much larger flux variations on significantly shorter time scales (of days or even hours), and could also show periodicity (e.g. due to eclipsing or pulsations), a behaviour not observed in AGN. Since some of our individual observations are separated by only several hours, it was possible to detect such extreme $\mathrm{X}$-ray variability on short time scales and use the generated X-ray light-curves, in combination with X-ray luminosity, spectra, and other wavelength identifications, to classify sources as XRBs.

Two XRBs have already been identified in PMH2004 with known binary systems in M 33: the black hole XRB X-8 (\#182) near the galactic nucleus, and the 3.4 day eclipsing HMXB X-7 (\#150). We classify eight additional sources as XRB candidates (\#41, 44, 75, 113, 134, 168, 180, and 254), mostly thanks to their light-curves.

The sources X-8 and X-7 are also variable in our data. The most luminous source $\mathrm{X}-8$ was found to change its flux by $10 \%$ with a period of 106 days (Dubus et al. 1997). Although this periodicity was not confirmed later, all other surveys of this source also suggest that it is variable (see Sect. 8). Our analysis of X-8 (Fig. B.3) shows flux variations of small amplitude (factor of 1.52) but high significance (35.4). As the light-curve shows, this small flux modulation occurs on a time scale of several hours.

The most accurate binary period of X-7 (3.45 days) has been determined by Pietsch et al. (2004b), using the individual XMM-Newton observations, together with Chandra and optical data. Our light-curve (Fig. B.2) also includes two XMM-Newton observations not analysed by Pietsch et al. (2004b). However, the source is still found only in high or low state, with the shortest separation between the states less than two days, and no transit into or out of eclipse was observed.

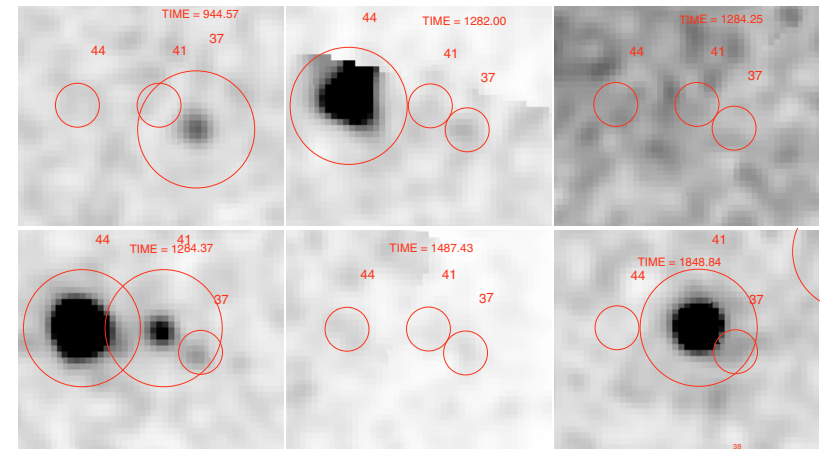

Fig. 11. The broad band XMM-Newton EPIC gray scale images of the observations 4(a), 5(a), 10(a), 11(a), 15(a), and 4(b) (from upper left to lower right, respectively) showing the regions around sources \#44, 41, and 37. The big circles mark the positions of the detected sources, while small circles mark the positions of the sources below the detection threshold. The intensity scale in all images is linear from 0 to $2.90472 \mathrm{ct} \mathrm{s}^{-1}$ pixel $^{-1}$. The observation times in days after JD 2450814.5 are marked on each image.

The shape of the light-curve of one XRB candidates (\#113) resembles that of X-7 (Fig. B.1). It shows the source in high and low states, with flux variations of a factor of at least 54 . This source is one of the two extended sources in our catalogue, identified as the HII region BCLMP 290 in SIMBAD, and it is also listed as an SNR by Gordon et al. (1999). According to its HRs, the source was classified as "hard" in the combined data catalogue. Inspection of archival Chandra images indicates that this complex region, which could not be resolved by XMM-Newton, contains different X-ray emitting objects. The light-curve shows that the transitions from low to high state occur in $\sim 0.25$ days.

Two other XRB candidates (\#44 and 41) are shown in Fig. 11. Source \#44 exhibits the highest variability in our sample, changing its flux by a factor of at least 144 . As the images in Fig. 11 and the corresponding light-curve (Fig. 9) show, the source is very strong (with a luminosity of $2 \times 10^{37} \mathrm{erg} \mathrm{s}^{-1}$ in the high state) on two images separated by $\sim 2.4$ days, but could not be detected in the other observations. Sources \#41 shows similar behaviour (Fig. B.1). The nearby source \#37 is classified as "hard", without significant variability.

As already noted, the complex HII region NGC 604 (\#254) has an SNR spectrum, but its light-curve (Fig. B.4) shows several transitions from low to high state in a fraction of a day, so we also included it in the list of the XRB candidates, together with sources 75,134 , and 180, which have similar temporal characteristics (Figs. B.1-B.3, respectively). Source \#75 was also detected as a variable source in earlier X-ray surveys (see Haberl \& Pietsch 2001, and references therein), and its luminosity is in the range expected for an XRB. Source \#180, detected only in the present catalogue, has been identified in SIMBAD as a peculiar object of unknown nature (Fabrika \& Sholukhova 1995). Finally, PMH2004 note several other objects as possible XRB candidates, because of their cross-correlation with variable stars. One of these sources (\#168) is included in our list of the XRB candidates, because of its high variability amplitude (Fig. B.2), spectral 


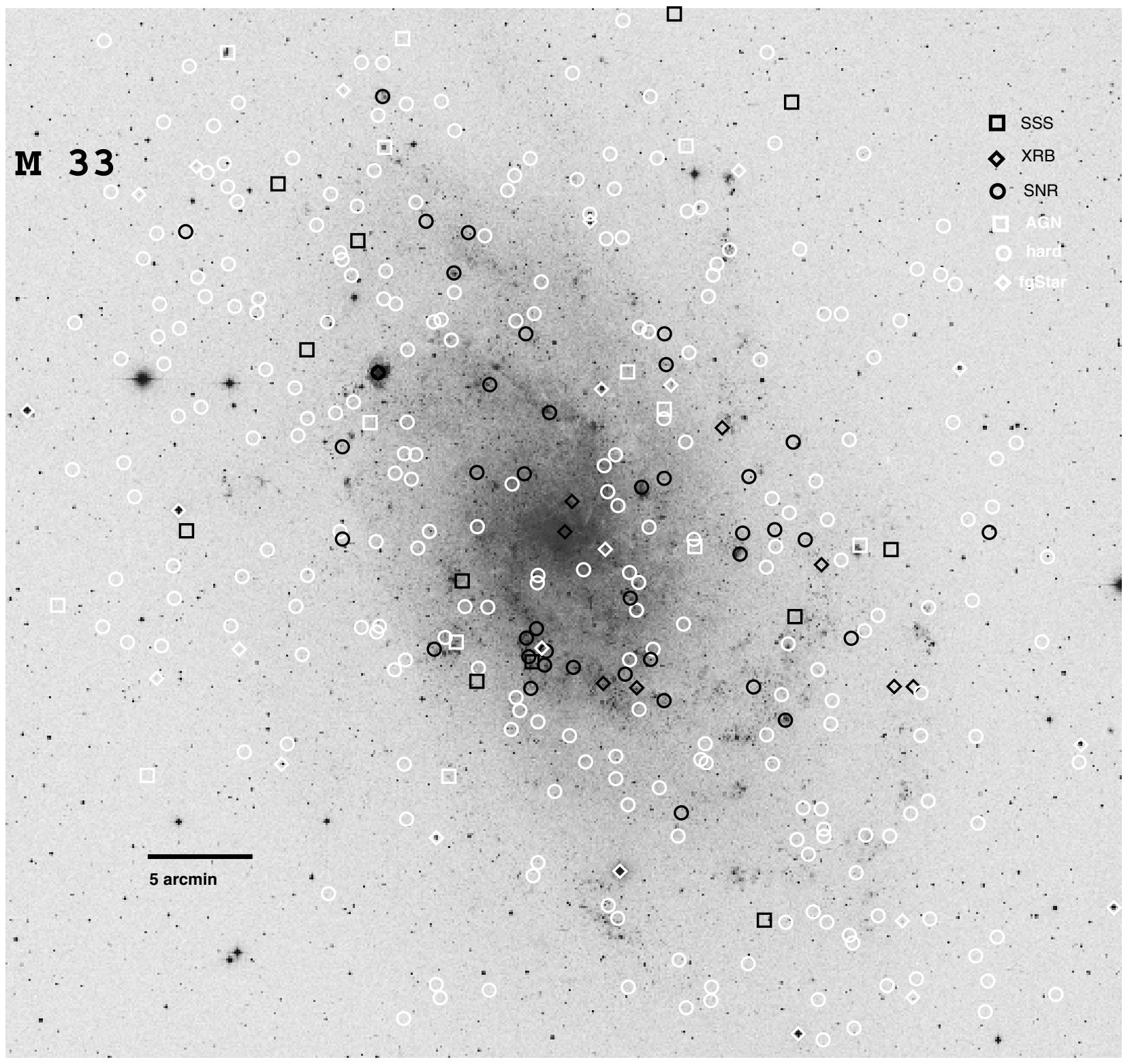

Fig. 12. Spatial distribution of all 374 classified X-ray sources detected in the direction of M 33 in both the combined and individual data catalogues.

characteristics, and luminosity. We also confirmed the X-ray variability of source \#277 (Fig. B.4), identified with a variable star in SIMBAD. However, since its variability amplitude (slightly greater than 2) could also be observed in an AGN, we classify this source as "hard".

\subsection{Spatial distribution}

Figure 12 shows the spatial distribution of all 375 X-ray sources detected in the direction of M 33 and classified in the present catalogue, and/or by $\mathrm{PMH} 2004$. The majority of the sources in both surveys are classified as "hard"; they are evenly distributed over the total field of view, and are probably AGN. Foreground stars and candidates are also distributed over the total surveyed region, and the optical counterparts of most of them show up as bright objects in the optical image.
However, in our analysis, we also significantly increased the number of the classified sources, which are most probably intrinsic to M33 (shown with black symbols in Fig. 12). In addition to around 40 SNRs and candidates, we now have a significant population of XRB (10) and SSS (11) candidates in M33. The spatial distribution of the XRB and SSS candidates detected here supports their classification - most of them seem to be associated with the galactic disk or spiral arms, except 2 SSS candidates that are further away from the central region and could be foreground objects. The figure also shows a region to the South of the M 33 nucleus (along the Southern spiral arm) with 8 SNRs and 1 SSS source concentrated very close to each other, which could indicate a region of a recent star-formation episode.

We also made use of a recent XMM-Newton survey of M31 by Pietsch et al. (2005b) to compare the spatial 


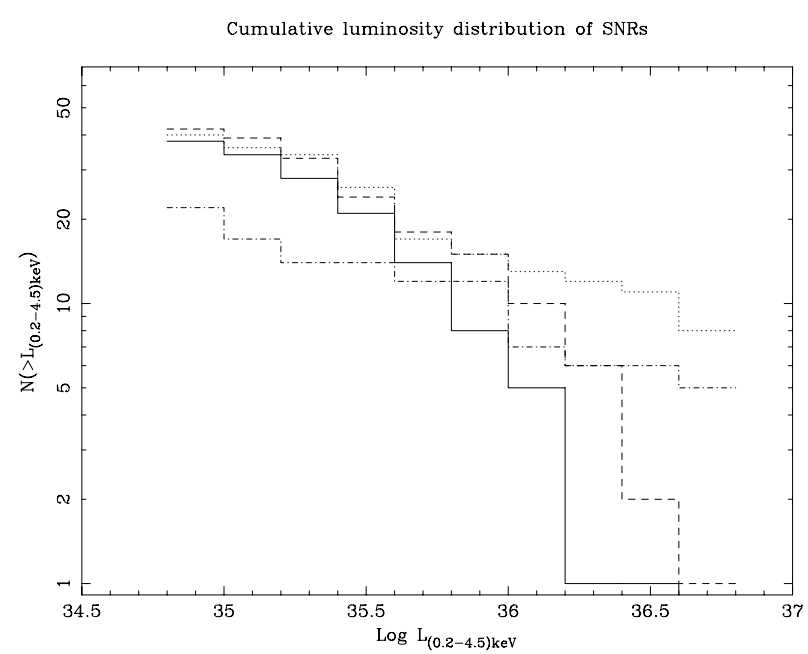

Fig. 13. The cumulative luminosity distribution of the SNRs and SNR candidates detected and classified in both XMM Newton catalogues of M 33 (this work and PMH2004, solid line), in the catalogue of M 31 (Pietsch et al. 2005b, dashed line), and in the ROSAT catalogues of LMC (Sasaki et al. 2000; Haberl \& Pietsch 1999, HRI dotted-dashed line; PSPC dotted line).

distribution of the detected X-ray sources in these two spiral galaxies. In contrast to M33, we clearly see a concentration of bright white and blue sources in the bulge of M31 (see Fig. 4 in Pietsch et al. 2005b). To compare this further, we selected all "white and blue" (i.e. "hard", XRBs and XRB candidates, AGN and AGN candidates) sources with luminosities above $\sim 3-4 \times 10^{36} \mathrm{erg} \mathrm{s}^{-1}$ (at that luminosity both surveys are complete over the whole area) in both catalogues - out of the 30 such sources in M33, 11 (or 37\%) are detected in the central observation, while most of the remaining sources are further away but within the $\mathrm{D}_{25}$ area. In M 31 the hard luminous sources are concentrated around the galactic centre - in a sample of 108 bright hard sources, we found a total of $71(66 \%)$ in the central observation.

\subsection{Luminosity distribution}

We present the luminosity distribution of the SNRs and XRBs in M 33 classified in both catalogues, and compare it with the same source populations detected in the XMM-Newton survey of M31 (Pietsch et al. 2005b). The catalogues are directly comparable, since they were derived using the same source detection procedures and classification. They also have similar sensitivity, the M31 survey covering approximately $1.2 \mathrm{deg}^{2}$ down to the (0.2-4.5) keV luminosity of $\sim 10^{35} \mathrm{erg} \mathrm{s}^{-1}$. Figure 13 shows the result of this comparison for the 38 SNRs and SNR candidates in M33, and 42 SNRs and candidates detected in M31 (Pietsch et al. 2005b). The distribution of SNRs in both spiral galaxies is almost the same, with slopes of $-23 \pm 3$ and $-24 \pm 2$ for M33 and M31, respectively. The Kolmogorov-Smirnov test gives a probability of $97.5 \%$ that the two distributions are derived from the same population. Although M 33 and M 31 reveal the same SNR luminosity distribution, there are many more SNRs in M33 per surveyed area detected in X-rays (the same number of detected SNRs in both galaxies, but the surveyed area of M 31 is almost 2 times larger).

Based on the multi-wavelength studies of the ISM in ours and the nearby galaxies Duric (2000), suggested that the reason for the intrinsically faint X-ray emission of SNRs in M31 could be a multi-phased ISM. Although the average gas density is relatively higher, SNRs are expanding into locally warm lowdensity regions. Previous studies suggest that the upper density limit of this ambient gas in M 31 is $0.1 \mathrm{~cm}^{-3}$ (Magnier et al. 1997). The similarity of the luminosity distribution of the detected SNRs in M 31 and M 33 seems to suggest that the ISM of M 33 also includes a low-density component.

The discrepancy between the relative number of bright SNRs in M 33 and the LMC, as found by Haberl \& Pietsch (2001) in the ROSAT analysis of M33, has been also recently discussed by Ghavamian et al. (2005). Ghavamian et al. (2005) use a sample of optically identified SNRs detected in Chandra and XMM-Newton data, and compare it with the SNRs detected in the ROSAT HRI survey (Sasaki et al. 2000). To check this result for our M33 SNR sample, we also plot (Fig. 13) 40 SNRs brighter than $\sim 10^{35} \mathrm{erg} \mathrm{s}^{-1}$ from the LMC PSPC catalogue (Haberl \& Pietsch 1999). We converted the ROSAT PSPC count rates to fluxes $\left(\mathrm{ECF}=2.17 \times 10^{-11} \mathrm{erg} \mathrm{cm}^{-2} \mathrm{ct}^{-1}\right)$ by using the same model to approximate the LMC source spectra that was used in the analysis of M33 and M31 (see Sect. 3). The figure shows that the SNRs in the LMC have a slightly flatter distribution (with a slope of $-18 \pm 3$ ) in agreement with the previous result, which was attributed to the relatively higher metallicity of the LMC (Haberl \& Pietsch 2001). Ghavamian et al. (2005) use a much smaller ROSAT HRI subsample of SNRs from Sasaki et al. (2000) for their comparison, which could be the reason for their slightly steeper LMC slope (also shown in Fig. 13).

The sample of the classified binaries and XRB candidates in M33 is still limited to performing detailed population studies, but it is now comparable to the relative number of XRBs detected in M 31 (Pietsch et al. 2005b). There are 7 XRB and 9 candidates in the M 31 catalogue, classified mainly from the optical cross-correlation and/or variability. However, there are 37 sources associated with the known globular clusters in M 31, most probably low mass X-ray binaries (LMXB). We could not detect any X-ray emission from the relatively smaller number of known globular clusters in M33 (Mochejska et al. 1998). Figure 14 shows the luminosity distribution of the XRBs detected in M 33 and M31. The globular cluster sources (LMXB) in M 31 are also shown. The distribution of the XRBs is clearly limited by the selection effect. It flattens just below the XID luminosity of $\sim 10^{37} \mathrm{erg} \mathrm{s}^{-1}$ in both galaxies, because the extreme variability used for the XRB classification could only be detected in highly luminous sources. The globular cluster sources, on the other hand, can be classified down to fainter luminosities, and have a slightly steeper distribution.

\section{Summary and conclusion}

We analysed all 24 XMM-Newton observations of M33 individually and compiled a catalogue of 350 point-like X-ray sources detected in those observations. A total of 39 of these 


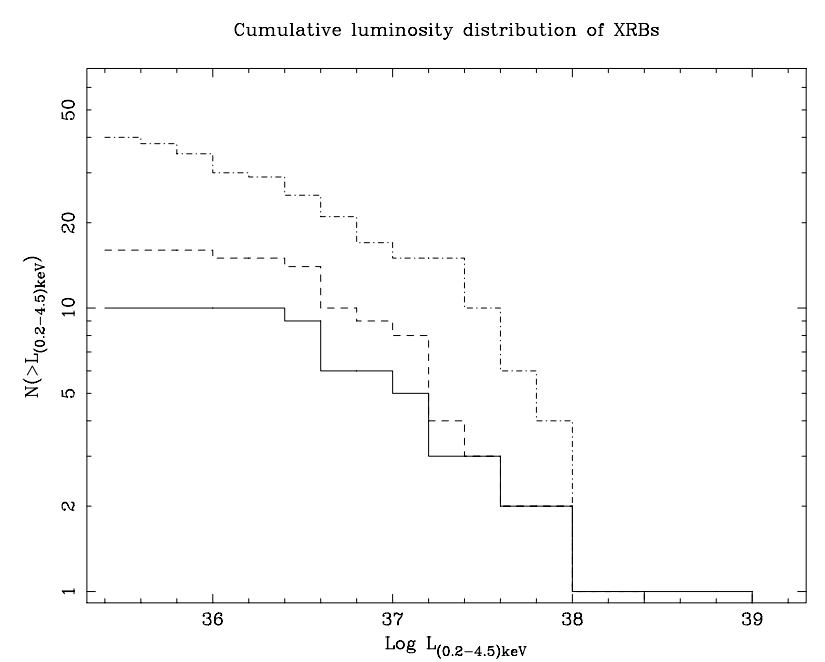

Fig. 14. The cumulative luminosity distribution of the XRB and XRB candidates detected and classified in both XMM Newton catalogues of M 33 (this work and PMH2004, solid line), and in the catalogue of M 31 (Pietsch et al. 2005b, XRBs - dashed line; globular cluster sources - dotted-dashed line).

objects were detected for the first time, while 311 had already been detected in PMH2004. For all sources we determined positions (improving on those reported in PMH2004) and the most relevant X-ray parameters such as XID fluxes, HRs, long-term variability, and correlations with other wavelength counterparts.

A total of 61 sources in our sample exhibit significant variability up to a factor of more than 144 on time scales from hours to months or years. As expected, the most variable sources in our sample have extremely soft or hard spectra, and we classified them as SSS and XRB candidates. There are also several foreground stars or candidates, along with AGN candidates exhibiting moderate flux variations (by a factor of approximately 2 to 6); but the majority of variable sources are "hard", probably unidentified XRBs or AGN. The brightest sources detected in our data (\#182 or X-8, \#131, \#112) show small flux variations detected with a high significance.

The variability amplitudes and shapes of the long-term light-curves were used, together with the HR method of PMH2004 and optical, infrared, and radio data, to classify all detected sources. In particular, we have used the light-curves to classify 8 new XRB candidates in M 33 that could not be distinguished from AGN using only the HR method. The detected variability was also used to reject the SNR classification of several sources. We also classified 11 SSS candidates, 7 of which are reported here for the first time. The detected and classified XRB and SSS candidates have significantly increased the $\mathrm{X}$-ray binary population in M 33 .

In addition, we classified 14 SNRs and 11 SNR candidates, 6 foreground stars and 23 star candidates, 12 AGN candidates, 1 galaxy, and 206 "hard" sources. The remaining 54 sources could not be classified using the proposed classification criteria. As already discussed by $\mathrm{PMH} 2004$, at least $50 \%$ of the "hard" and unclassified sources are background galaxies and
AGN, while the remaining 50-100 sources still await identification, most probably as XRBs or Crab-like SNRs within M 33.

The analysis of both the combined and individual observations of M33 have significantly increased the number of classified sources in M33. Using the deep combined image, it was possible to detect faint sources, in particular SNR and foreground star candidates. Approximately 50\% more of these sources were detected in the combined data than in the individual images. In addition, due to much better statistics for determining hardness ratios, the HR plots could be calibrated, and the classification method was established based, to a great extent, only on the X-ray spectral characteristics. In the analysis of the individual observations, on the other hand, it was possible to systematically search for long-term X-ray variability, and then use it as an efficient tool for classifying XRBs and other source classes. Furthermore, by analysing the individual images, it was possible to detect faint variable and transient sources such as SSS.

The spatial distribution of the detected sources in M 33 confirms that there is no pronounced bulge in this late-type spiral galaxy, in contrast to e.g. M31, where there is a clearly visible concentration of sources and diffuse emission around the galactic centre.

The relative contribution of the classified X-ray binary source population in M33 is now almost comparable to M 31 . Excluding the low mass XRBs associated with globular clusters, there are $7 \mathrm{XRB}$ and 9 candidates classified in a recent XMM-Newton survey of M 31 (Pietsch et al. 2005b). However, the number of classified XRB (10) and candidates in M 33 is still small, and more sensitive observations are needed to detect variability of fainter objects, and use it for source classification. In addition, optical follow-up observations are needed to confirm the XRB classifications.

There is approximately the same number of detected and classified SNRs and candidates in both galaxies, although the surveyed area of M31 and the total number of the detected sources are more than two times larger. The luminosity distribution of SNRs in both galaxies is similar, and has a slightly steeper slope than that of the LMC.

Acknowledgements. This publication makes use of: the USNOFS Image and Catalogue Archive operated by the United States Naval Observatory, Flagstaff Station (http://www .nofs . navy.mil/data/ fchpix/); data products from the Two Micron All Sky Survey, which is a joint project of the University of Massachusetts and the Infrared Processing and Analysis Center/California Institute of Technology, funded by the National Aeronautics and Space Administration and the National Science Foundation; the SIMBAD database, operated at CDS, Strasbourg, France; and the NASA/IPAC Extragalactic Database (NED) which is operated by the Jet Propulsion Laboratory, California Institute of Technology, under contract with the National Aeronautics and Space Administration. The compressed files of the "Palomar Observatory - Space Telescope Science Institute Digital Sky Survey" of the northern sky, based on scans of the Second Palomar Sky Survey, are copyright (c) 1993-1995 by the California Institute of Technology and are distributed herein by agreement. All Rights Reserved. Produced under Contract No. NAS 5-26555 with the National Aeronautics and Space Administration. The XMM-Newton project is supported by the Bundesministerium für Bildung und Forschung / Deutsches Zentrum für Luft- und Raumfahrt (BMBF/DLR), the 
Max-Planck Society, and the Heidenhain-Stiftung. Z.M. acknowledges support by the International Max Planck Research School on Astrophysics (IMPRS).

\section{References}

Boulesteix, J., Courtes, G., Laval, A., Monnet, G., \& Petit, H. 1974, A\&A, 37, 33

Brandt, W. N., Alexander, D. M., Hornschemeier, A. E., et al. 2001, AJ, 122, 2810

Condon, J. J., Cotton, W. D., Greisen, E. W., et al. 1998, AJ, 115, 1693

Cutri, R. M., Skrutskie, M. F., van Dyk, S., et al. 2003, VizieR Online Data Catalog, 2246

Di Stefano, R., Kong, A. K. H., Greiner, J., et al. 2004, ApJ, 610, 247

Dubus, G., \& Rutledge, R. E. 2002, MNRAS, 336, 901

Dubus, G., Charles, P. A., Long, K. S., \& Hakala, P. J. 1997, ApJ, 490, L47

Dubus, G., Charles, P. A., \& Long, K. S. 2004, A\&A, 425, 95

Duric, N. 2000, in Proc. 232. WE-Heraeus Seminar, 127

Fabrika, S., \& Sholukhova, O. 1995, Ap\&SS, 226, 229

Finlator, K., Ivezić, Ž., Fan, X., et al. 2000, AJ, 120, 2615

Freyberg, M. J., Briel, U. G., Dennerl, K., et al. 2004, in X-Ray and Gamma-Ray Instrumentation for Astronomy XIII, ed. K. A. Flanagan, \& O. H. W. Siegmund, Proc. SPIE, 5165, 112

Ghavamian, P., Blair, W. P., Long, K. S., et al. 2005, AJ, 130, 539

Gordon, S. M., Kirshner, R. P., Long, K. S., et al. 1998, ApJS, 117, 89

Gordon, S. M., Duric, N., Kirshner, R. P., Goss, W. M., \& Viallefond, F. 1999, ApJS, 120, 247

Greiner, J. 2000, New Astronomy, 5, 137

Greiner, J., Di Stefano, R., Kong, A., \& Primini, F. 2004, ApJ, 610, 261

Greiner, J., Hasinger, G., \& Kahabka, P. 1991, A\&A, 246, L17

Haberl, F., \& Pietsch, W. 1999, A\&AS, 139, 277

Haberl, F., \& Pietsch, W. 2001, A\&A, 373, 438

Haberl, F., Dennerl, K., \& Pietsch, W. 2003, A\&A, 406, 471

Hog, E., Kuzmin, A., Bastian, U., et al. 1998, A\&A, 335, L65

Ivanov, G. R., Freedman, W. L., \& Madore, B. F. 1993, ApJS, 89, 85

Jansen, F., Lumb, D., Altieri, B., et al. 2001, A\&A, 365, L1

Long, K. S., Dodorico, S., Charles, P. A., \& Dopita, M. A. 1981, ApJ, 246, L61
Long, K. S., Charles, P. A., Blair, W. P., \& Gordon, S. M. 1996, ApJ, 466, 750

Maccacaro, T., Gioia, I. M., Wolter, A., Zamorani, G., \& Stocke, J. T. 1988, ApJ, 326, 680

Magnier, E. A., Primini, F. A., Prins, S., van Paradijs, J., \& Lewin, W. H. G. 1997, ApJ, 490, 649

Markert, T. H., \& Rallis, A. D. 1983, ApJ, 275, 571

Mochejska, B. J., Kaluzny, J., Krockenberger, M., Sasselov, D. D., \& Stanek, K. Z. 1998, Acta Astronomica, 48, 455

Monet, D. G. 1998, BAAS, 30, 1427

Osborne, J. P., Borozdin, K. N., Trudolyubov, S. P., et al. 2001, A\&A, 378,800

Parmar, A. N., Sidoli, L., Oosterbroek, T., et al. 2001, A\&A, 368, 420

Peres, G., Reale, F., Collura, A., \& Fabbiano, G. 1989, ApJ, 336, 140

Pietsch, W., Ehle, M., Haberl, F., Misanovic, Z., \& Trinchieri, G. 2003, Astron. Nachr., 324, 85

Pietsch, W., Misanovic, Z., Haberl, F., et al. 2004a, A\&A, 426, 11 [PMH2004]

Pietsch, W., Mochejska, B. J., Misanovic, Z., et al. 2004b, A\&A, 413, 879

Pietsch, W., Fliri, J., Freyberg, M., et al. 2005a, A\&A, 442, 879

Pietsch, W., Freyberg, M., \& Haberl, F. 2005b, A\&A, 434, 483

Primini, F. A., Forman, W., \& Jones, C. 1993, ApJ, 410, 615

Regan, M. W., \& Wilson, C. D. 1993, AJ, 105, 499

Rosati, P., Tozzi, P., Giacconi, R., et al. 2002, ApJ, 566, 667

Sasaki, M., Haberl, F., \& Pietsch, W. 2000, A\&AS, 143, 391

Schulman, E., \& Bregman, J. N. 1995, ApJ, 441, 568

Skrutskie, M. F., Schneider, S. E., Stiening, R., et al. 1997, in The Impact of Large Scale Near-IR Sky Surveys, ASSL, Vol. 210, 25

Stark, A. A., Gammie, C. F., Wilson, R. W., et al. 1992, ApJS, 79, 77

Strüder, L., Briel, U., Dennerl, K., et al. 2001, A\&A, 365, L18

Trinchieri, G., Fabbiano, G., \& Peres, G. 1988a, ApJ, 325, 531

Trinchieri, G., Fabbiano, G., \& Peres, G. 1988b, ApJ, 329, 1037

Trümper, J., Hasinger, G., Aschenbach, B., Braeuninger, H., \& Briel, U. G. 1991, Nature, 349, 579

Turner, M. J. L., Abbey, A., Arnaud, M., et al. 2001, A\&A, 365, L27 van den Bergh, S. 1991, PASP, 103, 609

van den Heuvel, E. P. J., Bhattacharya, D., Nomoto, K., \& Rappaport, S. A. 1992, A\&A, 262, 97

Zaritsky, D., Elston, R., \& Hill, J. M. 1989, AJ, 97, 97 


\section{Online Material}




\section{Appendix A: Full band images}
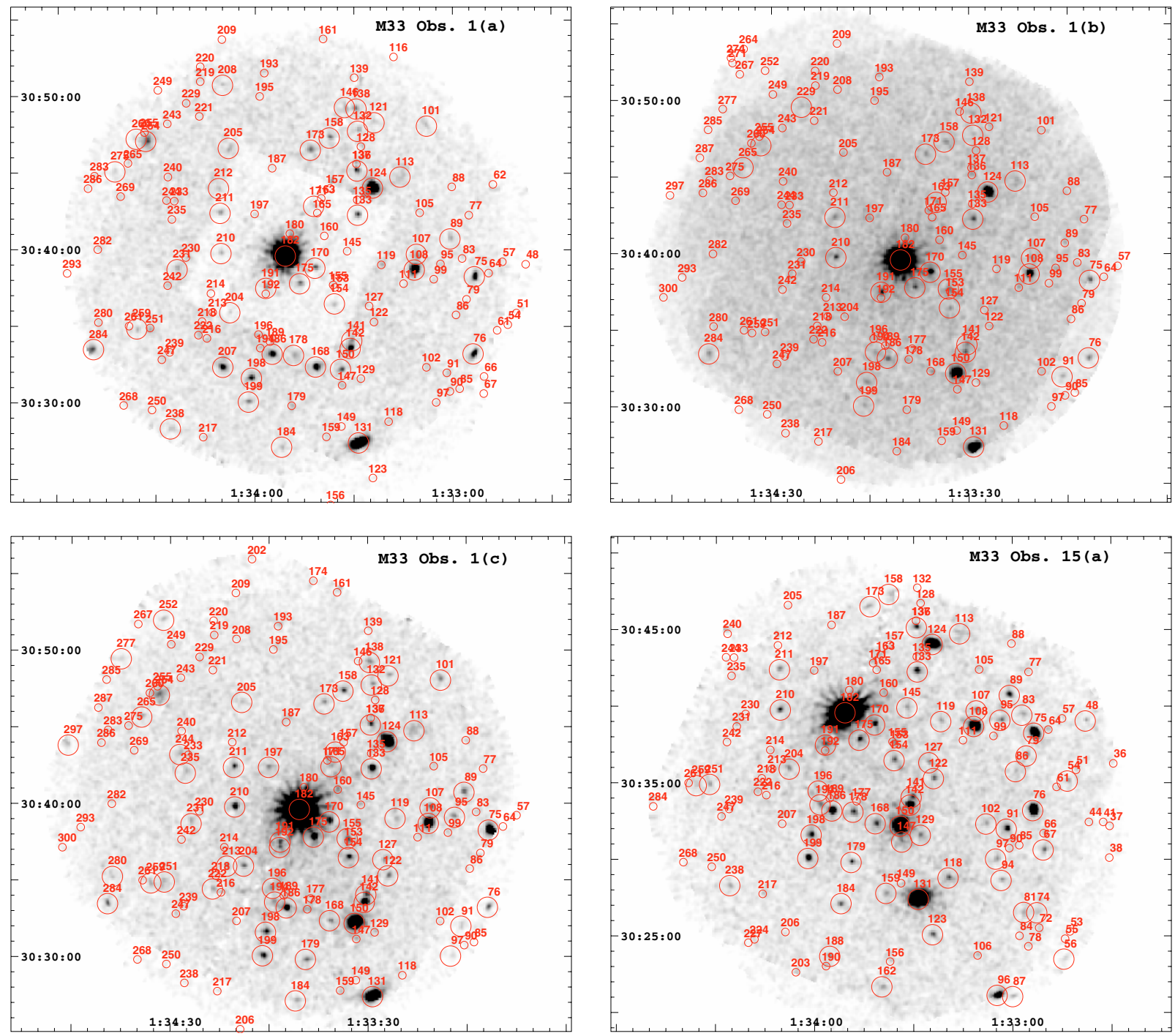

Fig. A.1. The broad band (0.2-12.0 keV) gray scale XMM-Newton EPIC images of the observations 1(a) (upper left), 1(b) (upper right), 1(c) (lower left), and 15(a) (lower right), combining PN, MOS1, and MOS2 data. The images are smoothed with a Gaussian of $F W H M 6^{\prime \prime}$. The intensity scale is linear from 0.00765 to $3.0785 \mathrm{ct} \mathrm{s}^{-1}$ pixel $^{-1}$ (upper left, lower right, lower left), and from 0.00765 to $6.7785 \mathrm{ct} \mathrm{s}^{-1} \mathrm{pixel}^{-1}$ (upper right). The pixel size is $4^{\prime \prime}$. The big circles represent the sources detected above the likelihood threshold of 7, while the small circles mark the positions of the sources that are below the detection threshold in that observation, for which we determine only the upper limits of the count rates (see text). The numbers correspond to the SRC Id from Table 4. 
Z. Misanovic et al.: XMM-Newton survey of the Local Group galaxy M33, Online Material p 3
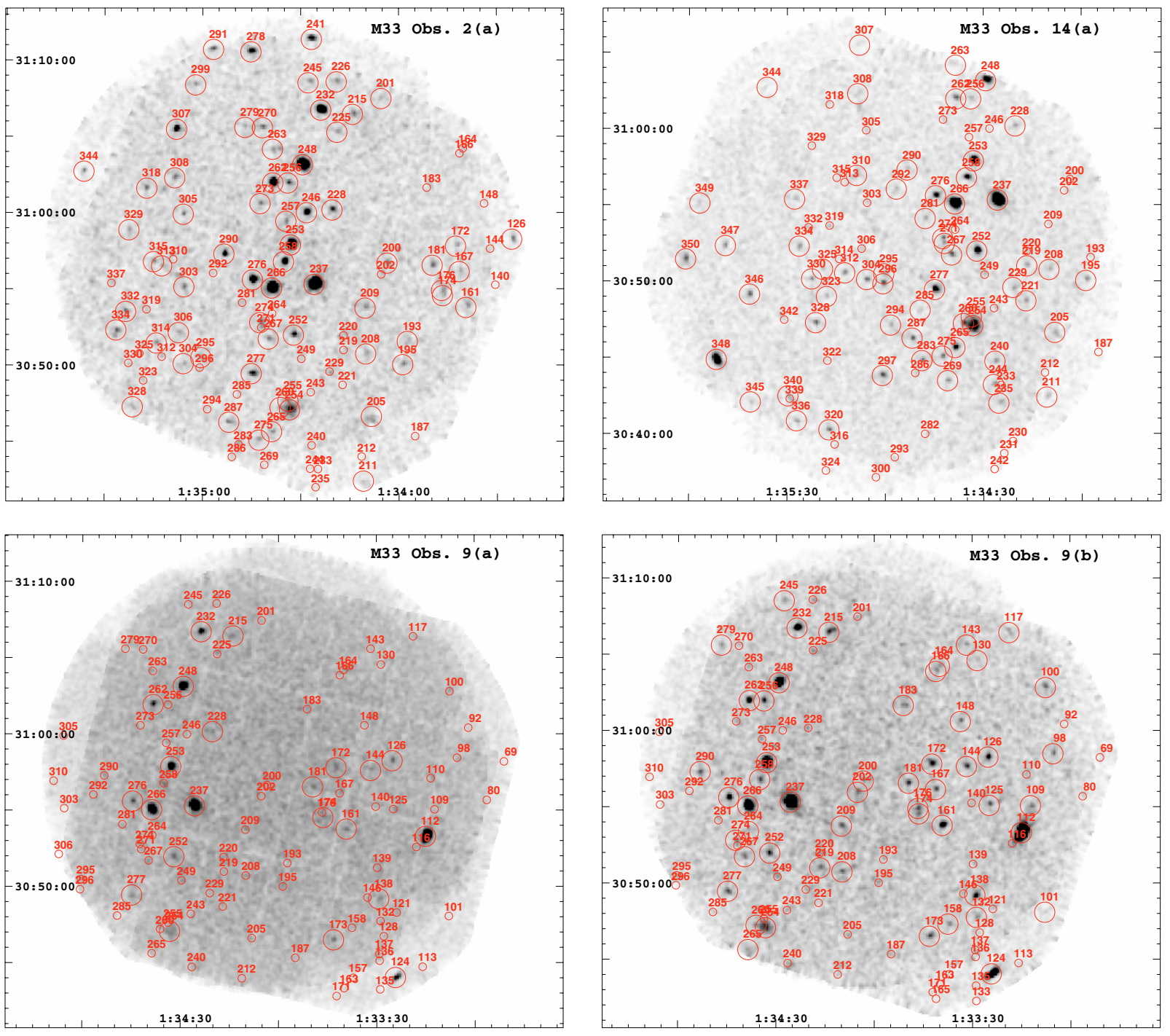

Fig. A.2. The broad band (0.2-12.0 keV) gray scale XMM-Newton EPIC images of the observations 2(a) (upper left), 14(a) (upper right), 9(a) (lower left), and 9(b) (lower right), combining PN, MOS1, and MOS2 data. The images are smoothed with a Gaussian of $F W H M 6^{\prime \prime}$. The intensity scale is linear from 0.00513 to $3.36775 \mathrm{ct} \mathrm{s}^{-1}$ pixel $^{-1}$ (upper left, upper right, lower right), and from 0.00513 to $8.2400775 \mathrm{ct} \mathrm{s}^{-1} \mathrm{pixel}^{-1}$ (lower left). The pixel size is $4^{\prime \prime}$. The big circles represent the sources detected above the likelihood threshold of 7, while the small circles mark the positions of the sources that are below the detection threshold in that observation, for which we determine only the upper limits of the count rates (see text). The numbers correspond to the SRC Id from Table 4. 
Z. Misanovic et al.: XMM-Newton survey of the Local Group galaxy M33, Online Material p 4
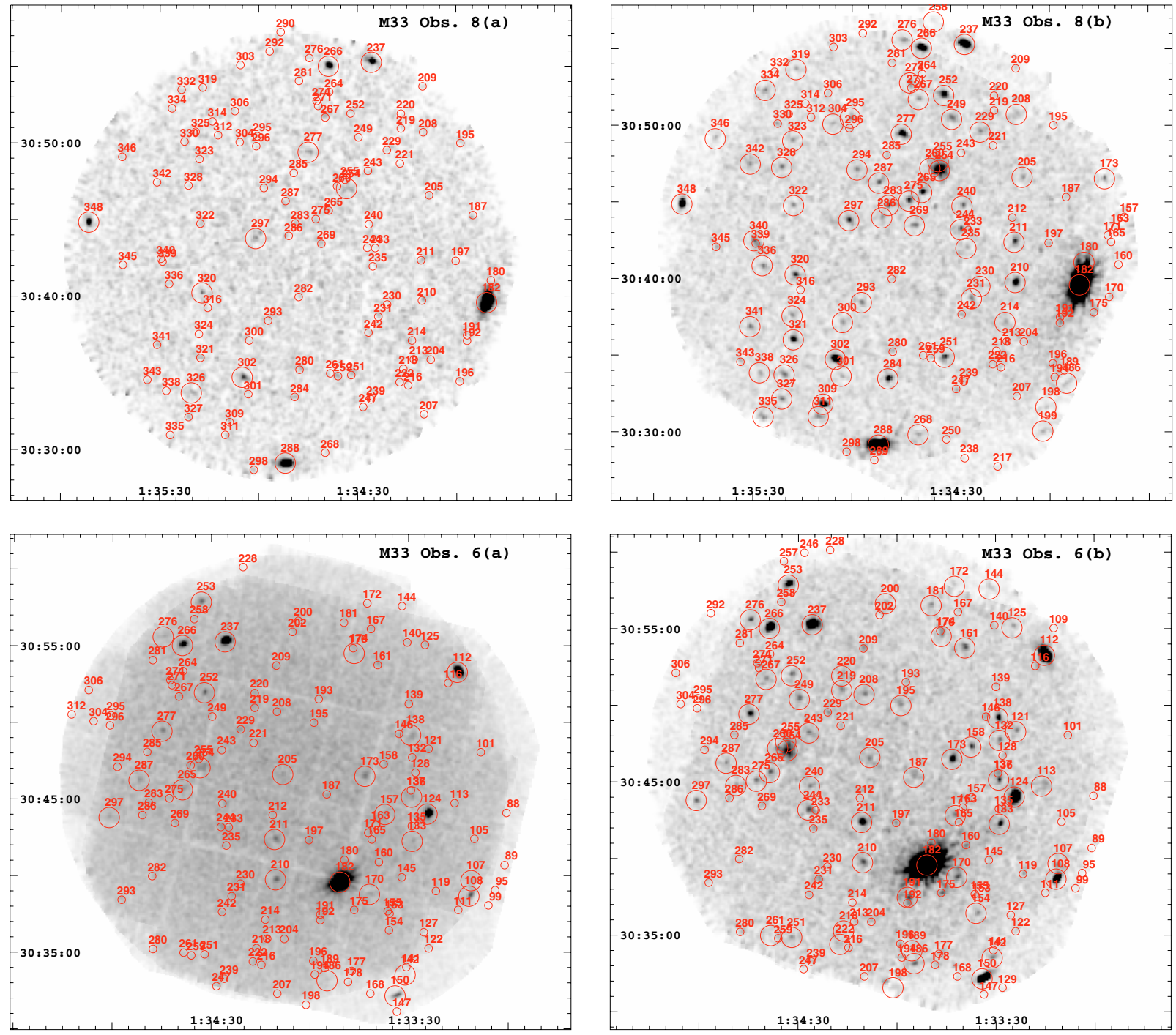

Fig. A.3. The broad band (0.2-12.0 keV) gray scale XMM-Newton EPIC images of the observations 8(a) (upper left), 8(b) (upper right), 6(a) (lower left), and 6(b) (lower right), combining PN, MOS1, and MOS2 data. The images are smoothed with a Gaussian of $F W H M$ 6". The intensity scale is linear from 0.00153 to 1.55803 (upper left), 0.001527 to 3.02803 (upper right), 0.001527 to 15.65901 (lower left), and from 0.001527 to $3.99803 \mathrm{ct} \mathrm{s}^{-1}$ pixel $^{-1}$ (lower right). The pixel size is $4^{\prime \prime}$. The big circles represent the sources detected above the likelihood threshold of 7, while the small circles mark the positions of the sources that are below the detection threshold in that observation, for which we determine only the upper limits of the count rates (see text). The numbers correspond to the SRC Id from Table 4. 
Z. Misanovic et al.: XMM-Newton survey of the Local Group galaxy M33, Online Material p 5
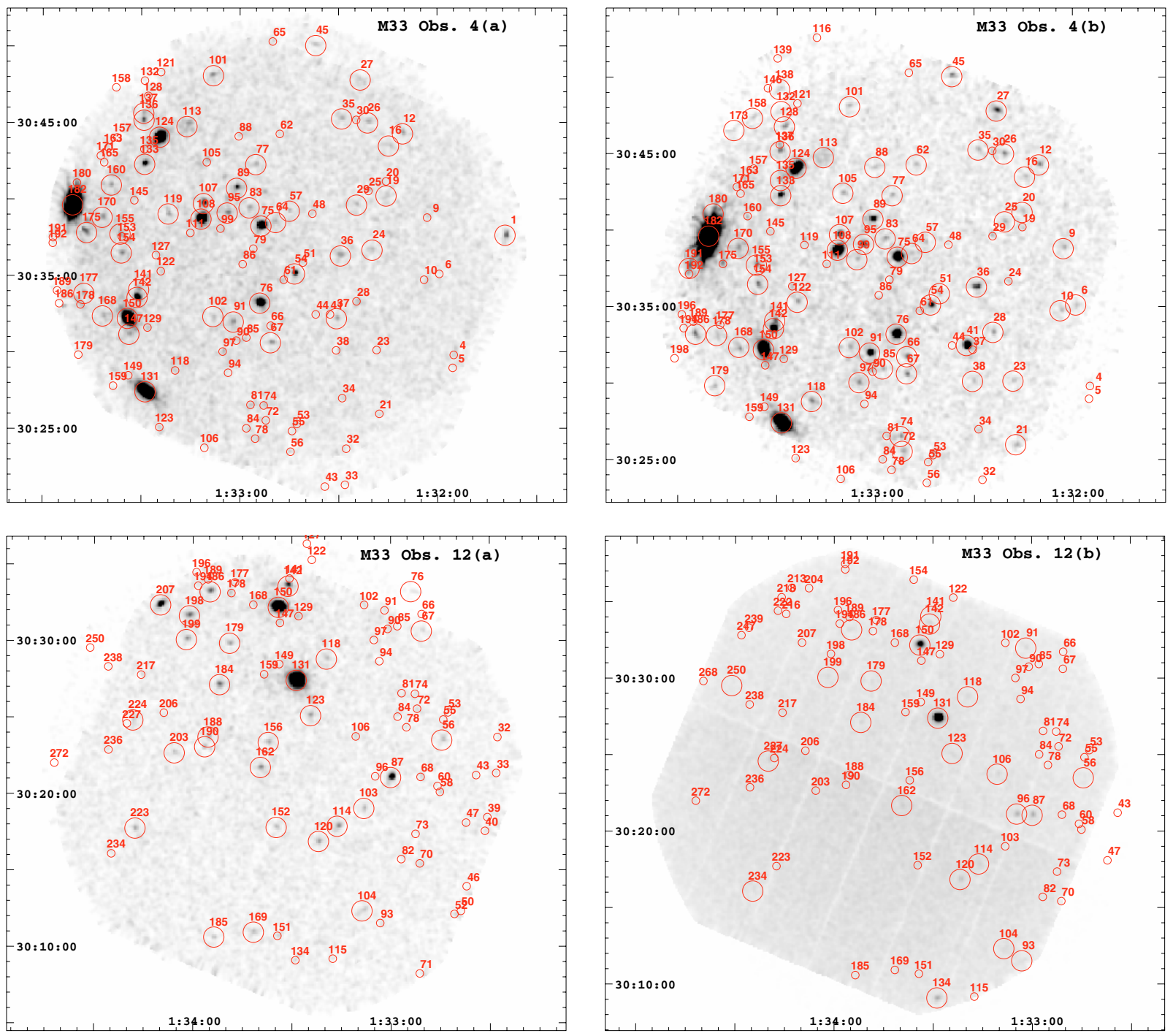

Fig. A.4. The broad band (0.2-12.0 keV) gray scale XMM-Newton EPIC images of the observations 4(a) (upper left), 4(b) (upper right), 12(a) (lower left), and 12(b) (lower right), combining PN, MOS1, and MOS2 data. The images are smoothed with a Gaussian of FWHM 6". The intensity scale is linear from 0.02276 to 4.18462 (upper left, upper right and lower left), and 0.02276 to $30.74765 \mathrm{ct} \mathrm{s}^{-1} \mathrm{pixel}^{-1}$ (lower right). The pixel size is $4^{\prime \prime}$. The big circles represent the sources detected above the likelihood threshold of 7 , while the small circles mark the positions of the sources that are below the detection threshold in that observation, for which we determine only the upper limits of the count rates (see text). The numbers correspond to the SRC Id from Table 4. 
Z. Misanovic et al.: XMM-Newton survey of the Local Group galaxy M33, Online Material p 6
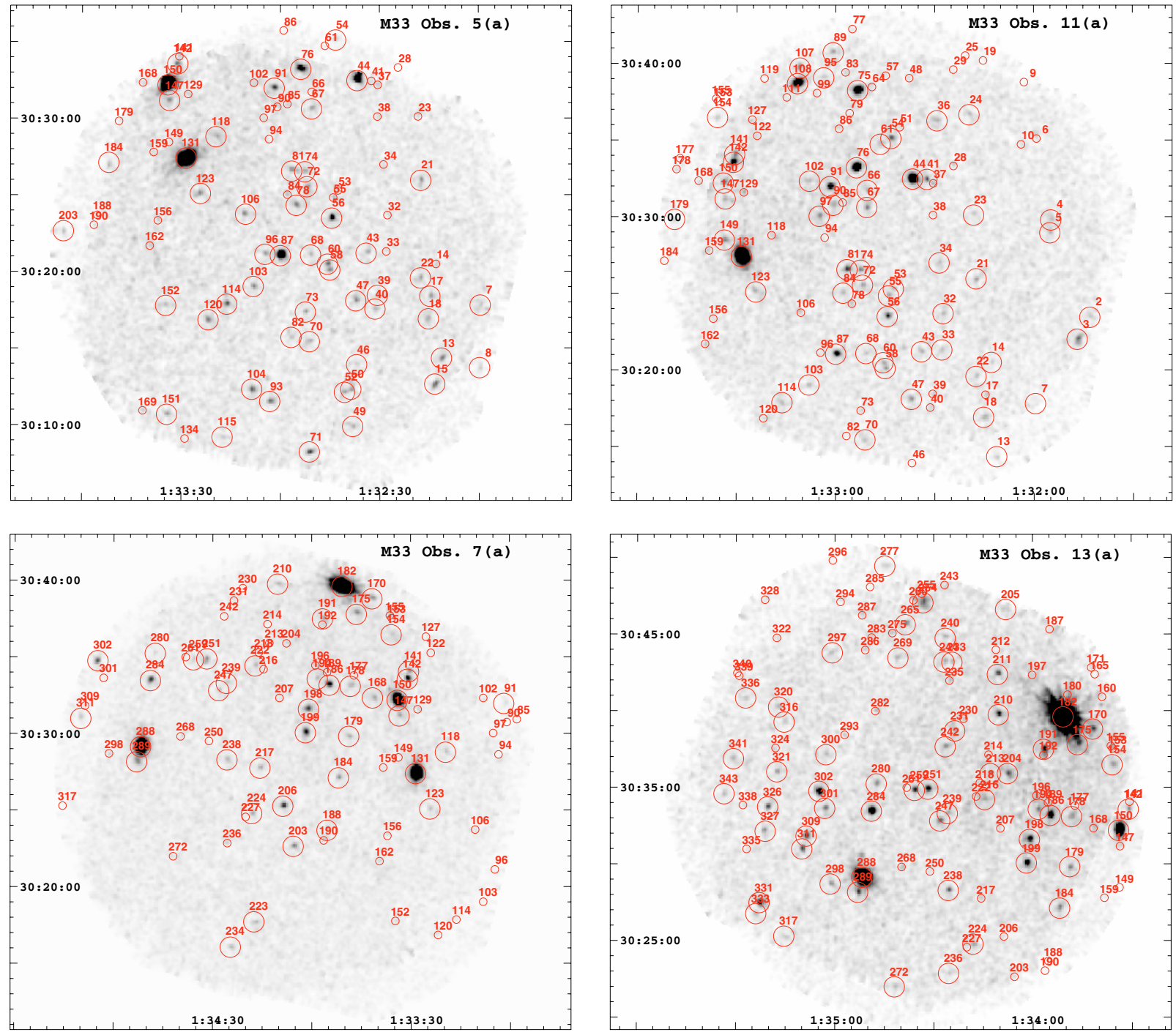

Fig. A.5. The broad band (0.2-12.0 keV) gray scale XMM-Newton EPIC images of the observations 5(a) (upper left), 11(a) (upper right), 7(a) (lower left), and 13(a) (lower right), combining PN, MOS1, and MOS2 data. The images are smoothed with a Gaussian of $F W H M 6^{\prime \prime}$. The intensity scale is linear from 0.02343 to $3.94374 \mathrm{ct} \mathrm{s}^{-1} \mathrm{pixel}^{-1}$. The pixel size is $4^{\prime \prime}$. The big circles represent the sources detected above the likelihood threshold of 7, while the small circles mark the positions of the sources that are below the detection threshold in that observation, for which we determine only the upper limits of the count rates (see text). The numbers correspond to the SRC Id from Table 4. 
Z. Misanovic et al.: XMM-Newton survey of the Local Group galaxy M33, Online Material $p 7$
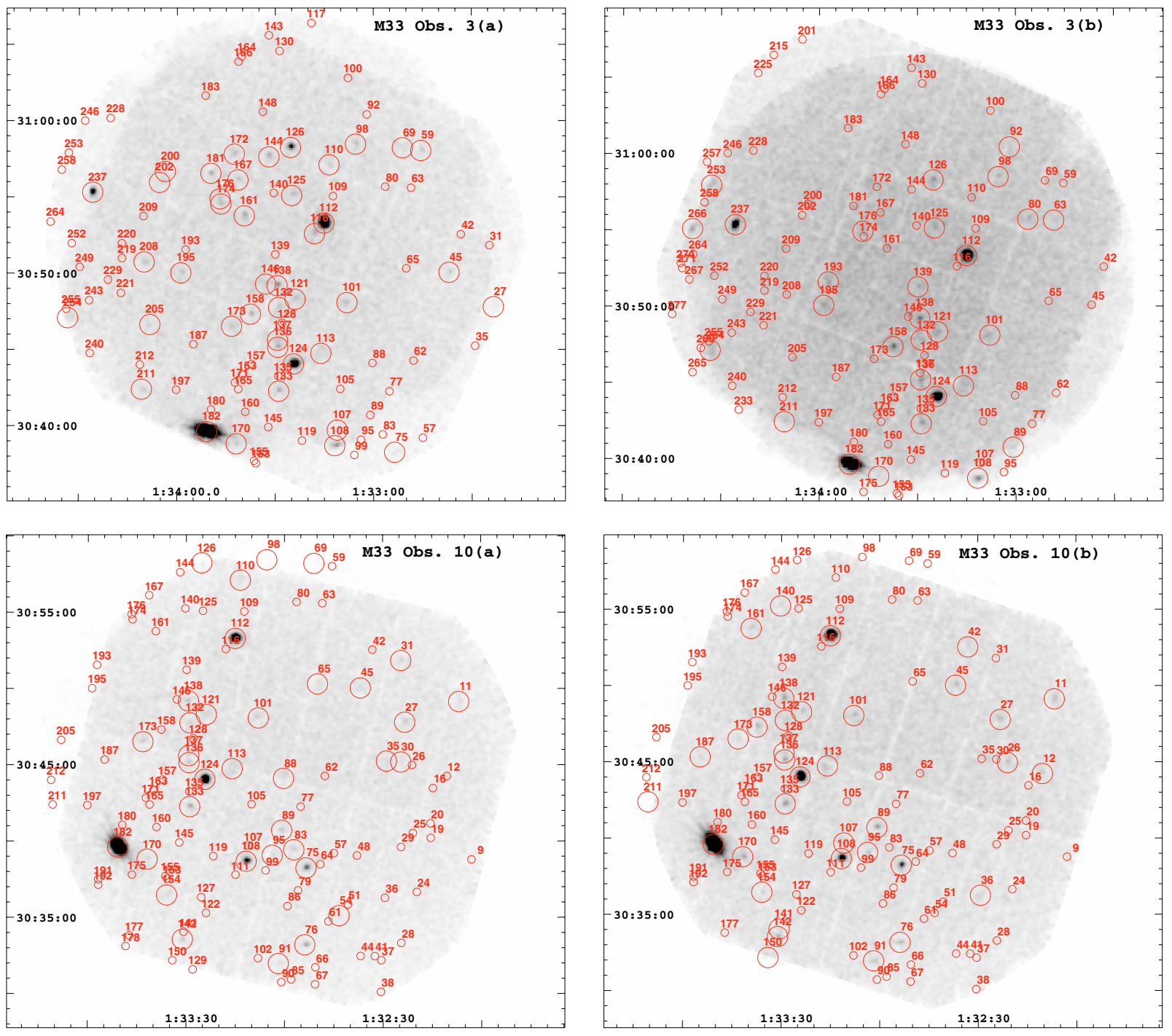

Fig. A.6. The broad band (0.2-12.0 keV) gray scale XMM-Newton EPIC images of the observations 3(a) (upper left), 3(b) (upper right), 10(a) (lower left), and 10(b) (lower right), combining PN, MOS1, and MOS2 data. The images are smoothed with a Gaussian of FWHM 6". The intensity scale is linear from 0.11473 to $15.81277 \mathrm{ct} \mathrm{s}^{-1} \mathrm{pixel}^{-1}$. The pixel size is $4^{\prime \prime}$. The big circles represent the sources detected above the likelihood threshold of 7, while the small circles mark the positions of the sources that are below the detection threshold in that observation, for which we determine only the upper limits of the count rates (see text). The numbers correspond to the SRC Id from Table 4. 
Z. Misanovic et al.: XMM-Newton survey of the Local Group galaxy M33, Online Material $p 8$

\section{Appendix B: light-curves of the individual sources}
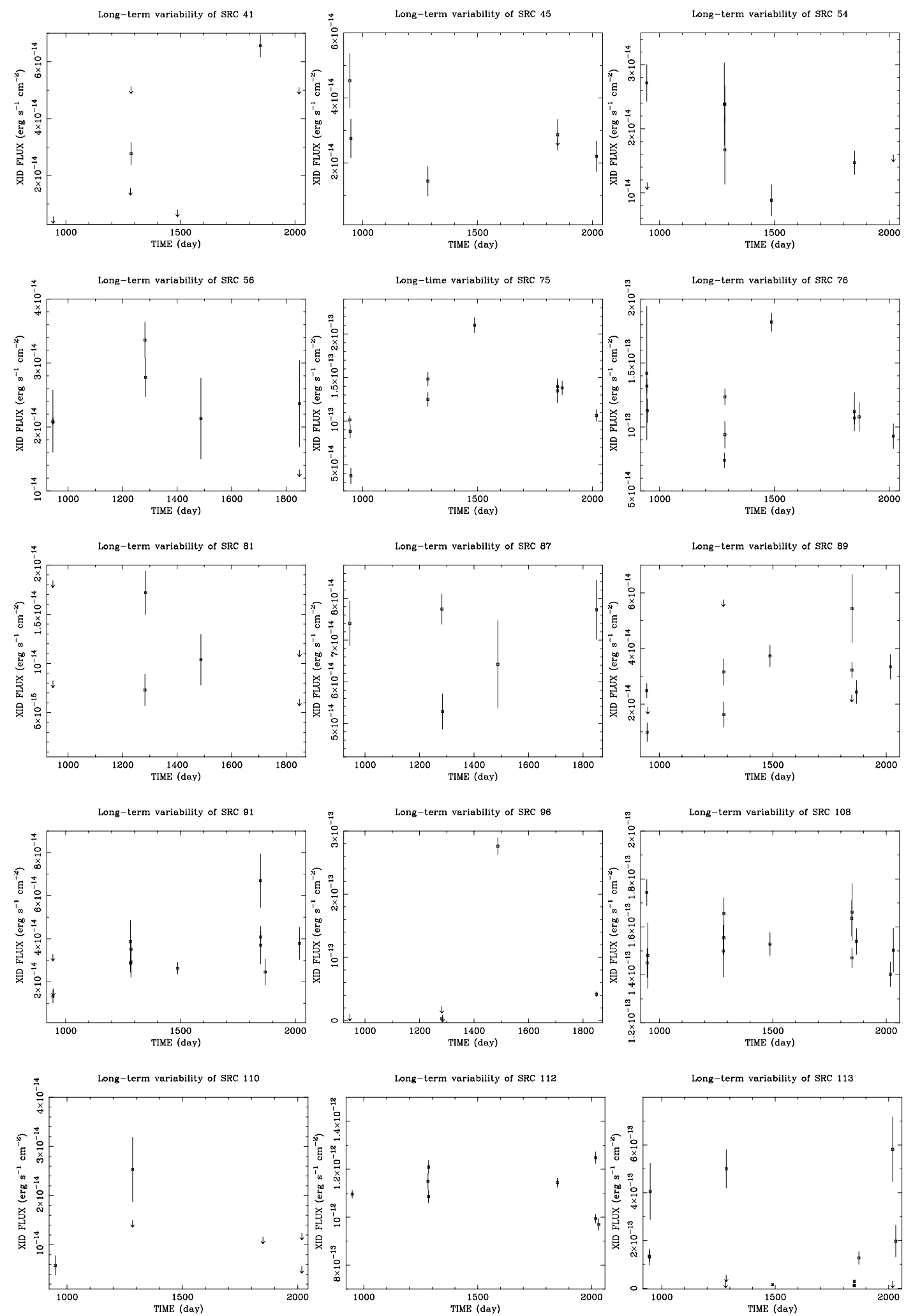

Fig. B.1. The long-term X-ray light-curves of sources \#41, \#45, \#54, \#56, \#75, \#76, \#81, \#87, \#89, \#91, \#96, \#108, \#110, \#112, and \#113. The downward arrows represent the $3 \sigma$ upper limits of the XID flux. The time is given in days after JD 2450 814.5. 
Z. Misanovic et al.: XMM-Newton survey of the Local Group galaxy M33, Online Material $p 9$
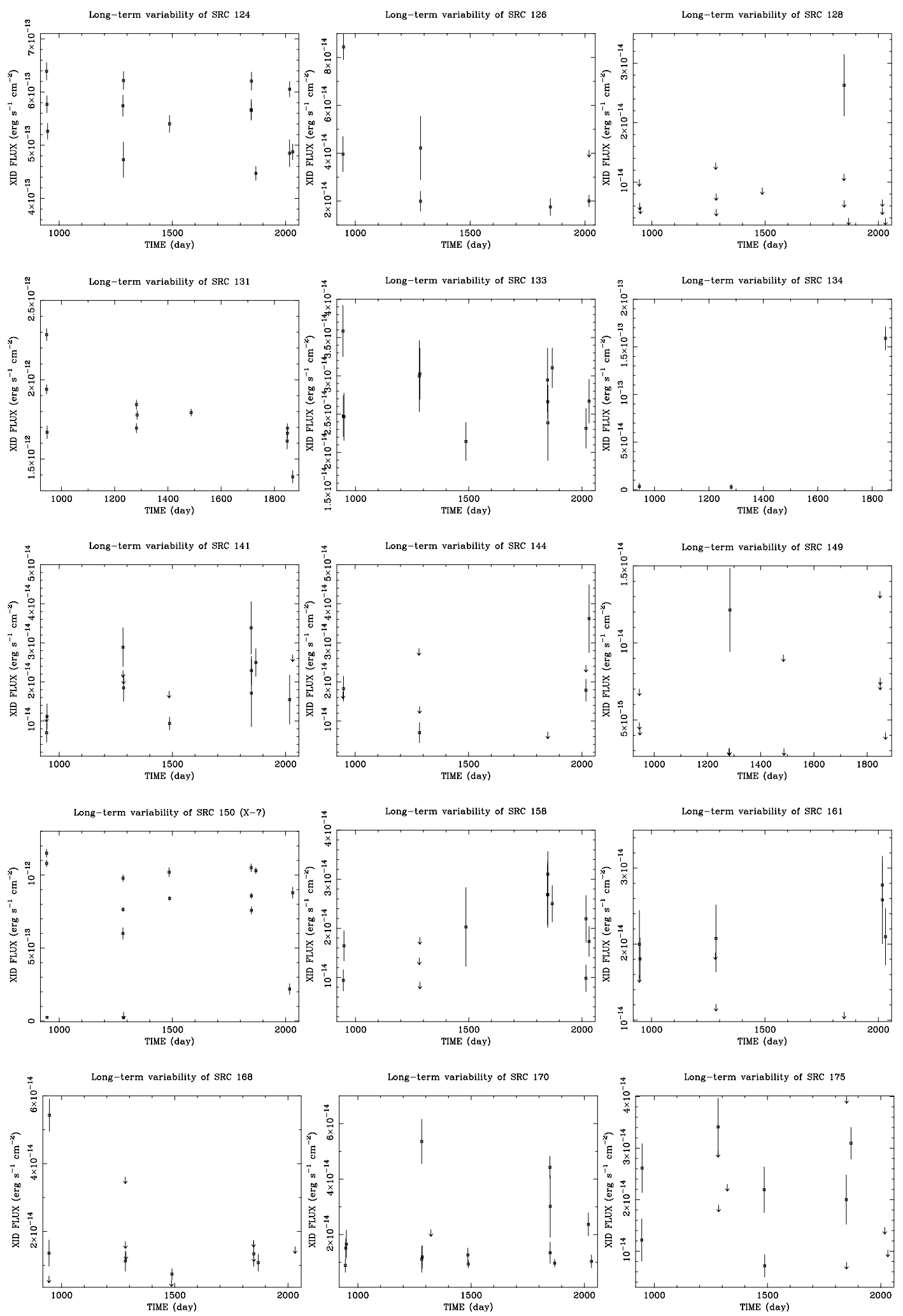

Fig. B.2. The long-term X-ray light-curves of sources \#124, \#126, \#128, \#131, \#133, \#134, \#141, \#144, \#149, \#150, \#158, \#161, \#168, \#170, and \#175. The downward arrows represent the $3 \sigma$ upper limits of the XID flux. The time is given in days after JD 2450 814.5. 
Z. Misanovic et al.: XMM-Newton survey of the Local Group galaxy M33, Online Material p 10
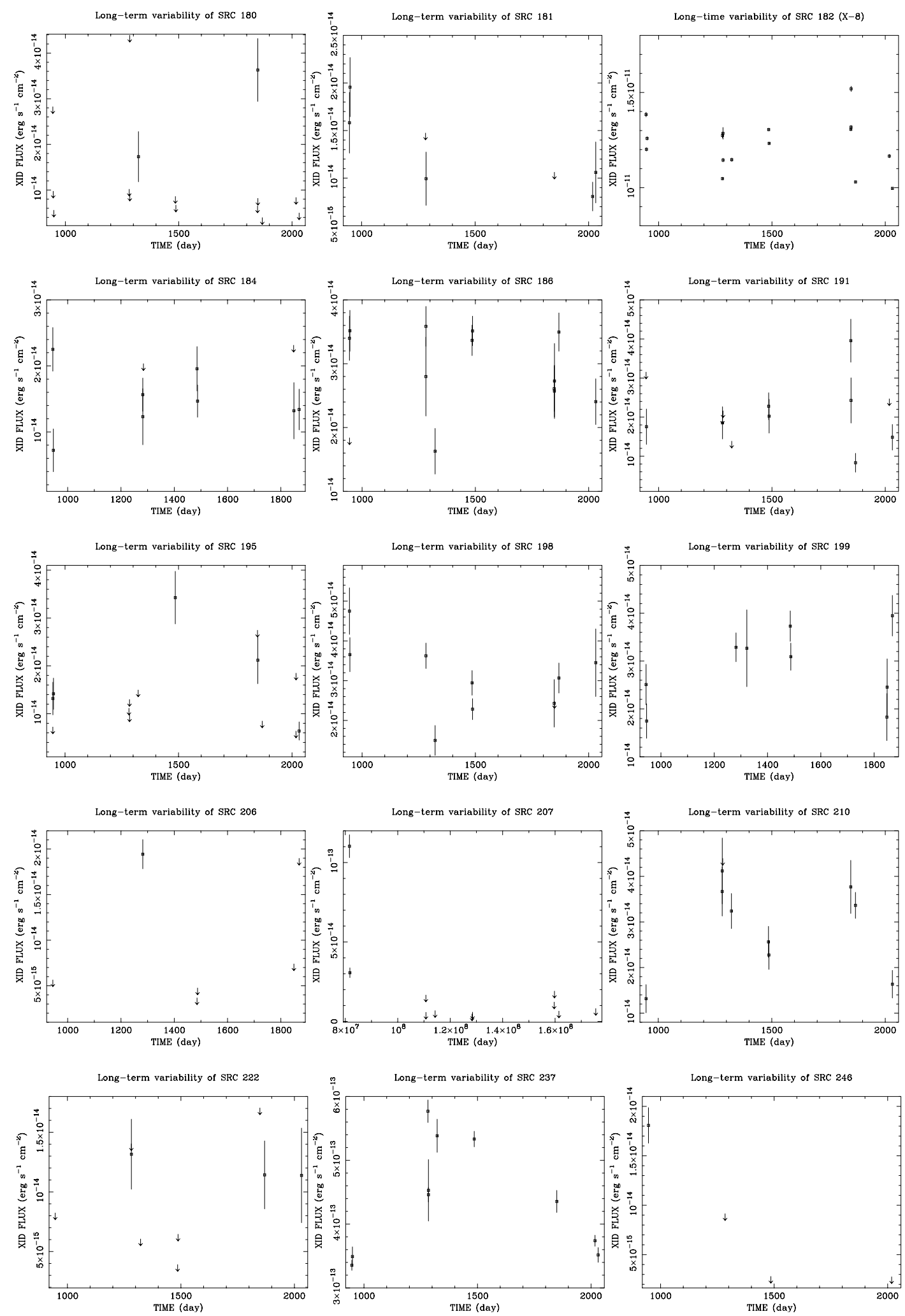

Fig. B.3. The long-term X-ray light-curves of sources \#180, \#181, \#182, \#184, \#186, \#191, \#195, \#198, \#199, \#206, \#207, \#210, \#222, \#237, and \#246. The downward arrows represent the $3 \sigma$ upper limits of the XID flux. The time is given in days after JD 2 450 814.5. 
Z. Misanovic et al.: XMM-Newton survey of the Local Group galaxy M33, Online Material p 11
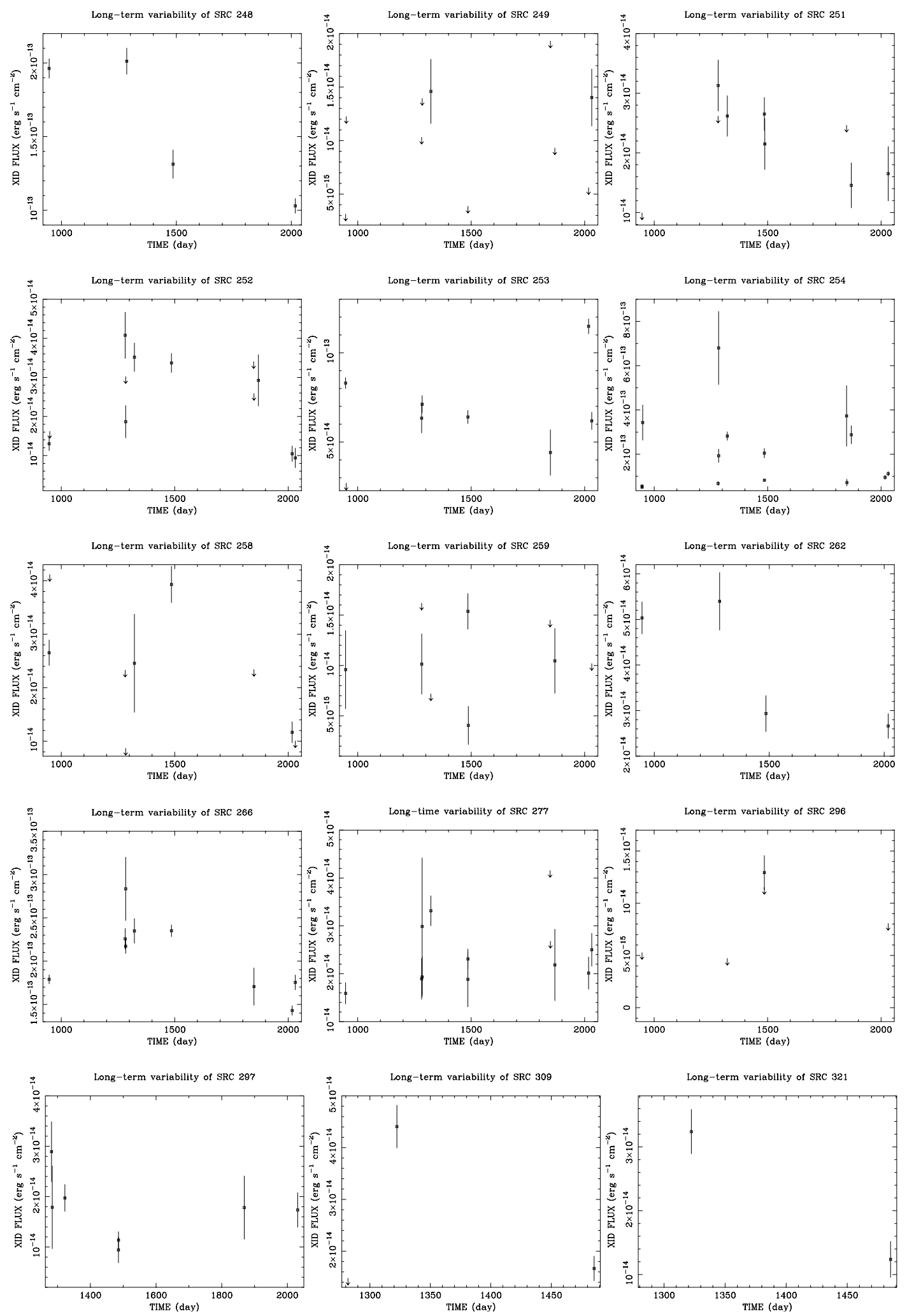

Fig. B.4. The long-term X-ray light-curves of sources \#248, \#249, \#251, \#252, \#253, \#254, \#258, \#259, \#262, \#266, \#277, \#296, \#297, \#309, and \#321. The downward arrows represent the $3 \sigma$ upper limits of the XID flux. The time is given in days after JD 2450 814.5. 